\title{
Infinite-Dimensional Calculus Under Weak Spatial Regularity of the Processes
}

\author{
Franco Flandoli ${ }^{1}$. Francesco Russo ${ }^{2}$. \\ Giovanni Zanco $^{3}$ (ID
}

Received: 7 November 2015 / Revised: 9 August 2016 / Published online: 25 November 2016

(C) The Author(s) 2016. This article is published with open access at Springerlink.com

\begin{abstract}
Two generalizations of Itô formula to infinite-dimensional spaces are given. The first one, in Hilbert spaces, extends the classical one by taking advantage of cancellations when they occur in examples and it is applied to the case of a group generator. The second one, based on the previous one and a limit procedure, is an Itô formula in a special class of Banach spaces having a product structure with the noise in a Hilbert component; again the key point is the extension due to a cancellation. This extension to Banach spaces and in particular the specific cancellation are motivated by path-dependent Itô calculus.
\end{abstract}

Keywords Stochastic calculus in Hilbert (Banach) spaces · Itô formula

Mathematics Subject Classification (2010) $60 \mathrm{H} 05 \cdot 60 \mathrm{H} 15 \cdot 60 \mathrm{H} 30$

\section{Introduction}

Stochastic calculus and in particular Itô formula have been extended since long time ago from the finite to the infinite-dimensional case. The final result in infinite dimensions is rather similar to the finite-dimensional one except for some important details. One of them is the fact that unbounded (often linear) operators usually appear in infi-

Giovanni Zanco

giovanni.zanco@ist.ac.at

1 Dipartimento di Matematica, Università di Pisa, Largo Bruno Pontecorvo 5, 56127 Pisa, Italy

2 ENSTA ParisTech, Université Paris-Saclay, Unité de Mathématiques appliquées, 828, boulevard des Maréchaux, 91120 Palaiseau, France

3 Institute of Science and Technology Austria (IST Austria), Am Campus 1, 3400 Klosterneuburg, Austria 
nite dimensions and Itô formula has to cope with them. If the Itô process $X(t)$, taking values in a Hilbert space $H$, satisfies an identity of the form

$$
\mathrm{d} X(t)=A X(t) \mathrm{d} t+B(t) \mathrm{d} t+C(t) \mathrm{d} W(t),
$$

where $A: D(A) \subset H \rightarrow H$ is an unbounded linear operator, then in the Itô formula for a functional $F:[0, T] \times H \rightarrow \mathbb{R}$ we have the term

$$
\left\langle A X(t), D_{x} F(t, X(t))\right\rangle,
$$

which requires $X(t) \in D(A)$ to be defined. The fact that $A X(t)$ also appears in (1) is not equally dramatic: Eq. (1) could be interpreted in weak or mild form, depending on the case. But the term (2) is less flexible. Sometimes it helps to interpret it as $\left\langle X(t), A^{*} D_{x} F(t, X(t))\right\rangle$ or similar reformulations, but this requires strong assumptions on $F$. Thus, in general, direct application of Itô formula is restricted to the case when $X(t) \in D(A)$. Among the ways to escape this difficulty let us mention the general trick to replace (1) by a regularized relation of the form $\mathrm{d} X_{n}(t)=A X_{n}(t) \mathrm{d} t+\cdots$ where $X_{n}(t) \in D(A)$ (the proof of Theorem 2.1 below is an example) and the socalled mild Itô formula proved in [6]. Another example of mild Itô formula under the assumptions that $D F \in D\left(A^{*}\right)$ was the object of [12], Theorem 4.8.

Less common but important for some classes of infinite-dimensional problems, for example the so-called path-dependent problems, is the phenomenon that $\frac{\partial F}{\partial t}(t, x)$ exists only when $x$ lives in a smaller space than $H$, for instance $D(A)$ (we shall clarify this issue in the examples of Sect. 5). And in Itô formula we have the term

$$
\frac{\partial F}{\partial t}(t, X(t))
$$

so again we need the condition $X(t) \in D(A)$.

The purpose of this paper is to give a generalization of Itô formula in infinitedimensional Hilbert and Banach spaces which solves the difficulties described above when the two terms

$$
\left\langle A X(t), D_{x} F(t, X(t))\right\rangle \text { and } \frac{\partial F}{\partial t}(t, X(t))
$$

compensate each other when they sum, although they are not well defined separately. This happens in a number of cases related to hyperbolic equations and path-dependent problems. This gives rise to a new Itô formula in which the term

$$
\frac{\partial F}{\partial t}(s, X(s))+\left\langle A X(s), D_{x} F(s, X(s))\right\rangle,
$$

that is a priori defined only when $X(s) \in D(A)$ is replaced by a term

$$
G(s, X(s))
$$

where $G(t, x)$ is an extension of $\frac{\partial F}{\partial t}(t, x)+\left\langle A x, D_{x} F(t, x)\right\rangle$. 
In this introduction, for simplicity of exposition, we have insisted on the formula in a Hilbert space $\mathrm{H}$, but one of the main purposes of our work is the further extension to a suitable class of Banach spaces, motivated by precise applications. Since the notations in the Banach case require more preliminaries, we address to Sect. 3 for this generalization.

Itô formulae for this kind of problems, both at the abstract level and in applications to path-dependent functionals, have been investigated by many authors; see $[3,4,9-$ $11,15]$; however, the idea to exploit the compensation explained above appears to be new and could be relevant for several applications.

Related to these problems is also the study of Kolmogorov equations in Banach spaces; see, for instance, $[2,13,14,16]$.

The paper is organized as follows. In Sect. 2 we give a first generalization of Itô formula in Hilbert spaces. It serves as a first step to prove a generalization to Banach spaces, described in Sect. 3. But this also applies directly to examples of hyperbolic SPDEs, as described in Sect. 4. Finally, in Sects. 5 and 6, we apply the extension to Banach spaces to several path-dependent problems: in Sect. 5 we consider typical path-dependent functionals; in Sect. 6 we deal with the important case when $F(t, x)$ satisfies an infinite-dimensional Kolmogorov equation.

\section{An Itô Formula in Hilbert Spaces}

Let $H, U$ be two separable Hilbert spaces (which will be always identified with their dual spaces) and $A: D(A) \subset H \rightarrow H$ be the infinitesimal generator of a strongly continuous semigroup $e^{t A}, t \geq 0$, in $H$. Let $(\Omega, \mathscr{F}, \mathbb{P})$ be a complete probability space, $\mathbb{F}=\left(\mathscr{F}_{t}\right)_{t \geq 0}$ be a complete filtration and $(W(t))_{t \geq 0}$ be a Wiener process in $U$ with nuclear covariance operator $Q$; we address to [7] for a detailed description of these notions of stochastic calculus in Hilbert spaces.

Let $B: \Omega \times[0, T] \rightarrow H$ be a progressively measurable process with $\int_{0}^{T}|B(s)| \mathrm{d} s<\infty$ a.s., $C: \Omega \times[0, T] \rightarrow L(U, H)$ be progressively measurable with $\int_{0}^{T}\|C(s)\|_{L(U, H)}^{2} \mathrm{~d} s<\infty$ a.s. and $X^{0}: \Omega \rightarrow H$ be a random vector, measurable w.r.t. $\mathscr{F}_{0}$; here $L(U, H)$ denotes the space of bounded linear operators from $U$ to $H$, with the corresponding norm $\|\cdot\|_{L(U, H)}$.

Let $X=(X(t))_{t \in[0, T]}$ be the stochastic process in $H$ defined by

$$
X(t)=e^{t A} X^{0}+\int_{0}^{t} e^{(t-s) A} B(s) \mathrm{d} s+\int_{0}^{t} e^{(t-s) A} C(s) \mathrm{d} W(s),
$$

formally solution to the equation

$$
\mathrm{d} X(t)=A X(t) \mathrm{d} t+B(t) \mathrm{d} t+C(t) \mathrm{d} W(t), \quad X(0)=X^{0} .
$$

We are interested here in examples where $X(s) \notin D(A)$ and also, for a given function $F:[0, T] \times H \rightarrow \mathbb{R}$, the derivative $\frac{\partial F}{\partial s}(s, x)$ exists only for a.e. $s$ and for $x \in D(A)$. In these cases the two terms $\frac{\partial F}{\partial s}(s, X(s))$ and $\langle A X(s), D F(s, X(s))\rangle$ have no meaning, in general. 
However, there are examples where the sum $\frac{\partial F}{\partial s}(s, X(s))+\langle A X(s), D F(s, X(s))\rangle$ has a meaning even if the two addends do not, separately. This paper is devoted to this class of examples.

We assume that there exists a Banach space $\widetilde{E}$ continuously embedded in $H$ such that

(I) $D(A) \subset \widetilde{E}$;

(II) $e^{t A}$ is strongly continuous in $\widetilde{E}$;

(III) $X(t) \in \widetilde{E}$;

(IV) almost surely the set $\{X(t)\}_{t \in[0, T]}$ is relatively compact in $\widetilde{E}$.

The space $\widetilde{E}$ can possibly coincide with the whole space $H$, but in general it is a smaller space endowed with a finer topology and it is not required to be a inner product space.

In the setting described above, our abstract result is the following one.

Theorem 1 Let $F \in C([0, T] \times H ; \mathbb{R})$ be twice differentiable with respect to its second variable, with $D F \in C([0, T] \times H ; H)$ and $D^{2} F \in C([0, T] \times H ; L(H, H))$, and assume the time derivative $\frac{\partial F}{\partial t}(t, x)$ exists for $(t, x) \in \mathscr{T} \times D(A)$ where $\mathscr{T} \subset[0, T]$ has Lebesgue measure $\lambda(\mathscr{T})=T$ and does not depend on $x$. Assume, moreover, that there exists a continuous function $G:[0, T] \times \widetilde{E} \rightarrow \mathbb{R}$ such that

$$
G(s, x)=\frac{\partial F}{\partial s}(s, x)+\langle A x, D F(s, x)\rangle \quad \text { for all }(t, x) \in \mathscr{T} \times D(A) .
$$

Let $X$ be the process defined in (3). Then

$$
\begin{aligned}
F(t, X(t))= & F\left(0, X^{0}\right)+\int_{0}^{t} G(s, X(s)) \mathrm{d} s \\
& +\int_{0}^{t}\left(\langle B(s), D F(s, X(s))\rangle+\frac{1}{2} \operatorname{Tr}\left(C(s) Q C(s)^{*} D^{2} F(s, X(s))\right)\right) \mathrm{d} s \\
& +\int_{0}^{t}\langle D F(s, X(s)), C(s) \mathrm{d} W(s)\rangle,
\end{aligned}
$$

where $D F$ and $D^{2} F$ denote the first and second Fréchet differentials of $F$ with respect to its second variable (the same notation will be used everywhere in this article).

For the proof we need a preliminary result, namely a "traditional" Itô formula that holds when $F$ is smooth.

Proposition 1 Let $\beta: \Omega \times[0, T] \rightarrow H$ and $\theta: \Omega \times[0, T] \rightarrow L(U, H)$ be two progressively measurable processes such that $|\beta(s)|$ and $\|\theta(s)\|_{L(U, H)}^{2}$ are integrable on $[0, T]$ a.s.; consider the Itô process $Z$ in $H$ given by

$$
Z(t)=Z^{0}+\int_{0}^{t} \beta(s) \mathrm{d} s+\int_{0}^{t} \theta(s) \mathrm{d} W(s) .
$$


If $F \in C^{1,2}([0, T] \times H)$ the following identity holds (in probability):

$$
\begin{aligned}
F(t, X(t))= & F\left(0, X^{0}\right)+\int_{0}^{t} \frac{\partial F}{\partial s}(s, X(s)) \mathrm{d} s \\
& +\int_{0}^{t}\left(\langle\beta(s), D F(s, X(s))\rangle+\frac{1}{2} \operatorname{Tr}\left(\theta(s) Q \theta(s)^{*} D^{2} F(s, X(s))\right)\right) \mathrm{d} s \\
& +\int_{0}^{t}\langle D F(s, X(s)), \theta(s) \mathrm{d} W(s)\rangle .
\end{aligned}
$$

Proof According to [10] we have that

$$
\begin{aligned}
F(t, X(t))= & F(0, X(0))+\int_{0}^{t}\left\langle D F(s, X(s)), \mathrm{d}^{-} X(s)\right\rangle \\
& +\int_{0}^{t} \frac{\partial F}{\partial t}(s, X(s)) \mathrm{d} s+\frac{1}{2} \int_{0}^{t} D^{2} F(s, X(s)) \mathrm{d} \widetilde{[X, X]}(s),
\end{aligned}
$$

where $\mathrm{d}^{-} X$ denotes the integral via regularization introduced in [10]. We remark that $\widetilde{[X, X]}$ is here the global quadratic variation of the process in (3).

By Theorem 3.6 and Proposition 3.8 of [12] we get

$$
\begin{aligned}
\int_{0}^{t}\left\langle D F(s, X(s)), \mathrm{d}^{-} X(s)\right\rangle= & \int_{0}^{t}\langle D F(s, X(s)), C(s) \mathrm{d} W(s)\rangle \\
& +\int_{0}^{t}\langle D F(s, X(s)), A X(s)+B(s)\rangle \mathrm{d} s .
\end{aligned}
$$

By Section 3.3 in [8]

$$
[X, X]^{\mathrm{d} z}(t)=\int_{0}^{t} C(s) Q^{1 / 2}\left(C(s) Q^{1 / 2}\right)^{*} \mathrm{~d} s,
$$

where $[X, X]^{\mathrm{d} z}$ is the Da Prato-Zabczyk quadratic variation; hence, proposition 6.12 of [8] implies that

$$
\int_{0}^{t} D^{2} F(s, X(s)) \mathrm{d} \widetilde{[X, X]}(s)=\int_{0}^{t} \operatorname{Tr}\left[D^{2} F(s, X(s)) C(s) Q^{1 / 2}\left(C(s) Q^{1 / 2}\right)^{*}\right] \mathrm{d} s .
$$

This concludes the proof.

Proof of Theorem 1 Let $\left\{\rho_{\varepsilon}\right\}_{\varepsilon \in(0,1]}, \rho_{\varepsilon}: \mathbb{R} \rightarrow \mathbb{R}$, be a family of mollifiers with $\operatorname{supp}\left(\rho_{\varepsilon}\right) \subseteq[0,1]$ for every $\varepsilon$. For $x \in H$ set $F(t, x)=F(0, x)$ if $t \in[-1,0)$ and $F(t, x)=F(T, x)$ if $t \in(T, T+1]$.

Denote by $J_{n}$ the Yosida approximations $J_{n}=n(n-A)^{-1}: H \rightarrow D(A)$, defined for every $n \in \mathbb{N}$, which satisfy $\lim _{n \rightarrow \infty} J_{n} x=x$ for every $x \in H$. One also has $\lim _{n \rightarrow \infty} J_{n}^{*} x=x, \lim _{n \rightarrow \infty} J_{n}^{2} x=x$ and $\lim _{n \rightarrow \infty}\left(J_{n}^{2}\right)^{*} x=x$ for every $x \in H$, used several times below, along with the fact that the operators $J_{n}$ and $J_{n}^{*}$ are equibounded. 
All these facts are well known and can be found also in [7]. Moreover, it is easy to show that the family $J_{n}^{2}$ converges uniformly on compact sets to the identity (in the strong operator topology). Since $A$ generates a strongly continuous semigroup in $\widetilde{E}$ as well, all the properties of $J_{n}$ and $J_{n}^{2}$ just listed hold also in $\widetilde{E}$ (with respect to its topology).

Define now $F_{\varepsilon, n}:[0, T] \times H \rightarrow \mathbb{R}$ as

$$
F_{\varepsilon, n}(t, x)=\left(\rho_{\varepsilon} * F\left(\cdot, J_{n} x\right)\right)(t)
$$

It is not difficult to show that $F_{\varepsilon, n} \in C^{1,2}([0, T] \times H ; \mathbb{R})$. Notice also that

$$
\begin{aligned}
\frac{\partial F_{\varepsilon, n}}{\partial t}(t, x) & =\left(\dot{\rho}_{\varepsilon} * F\left(\cdot, J_{n} x\right)\right)(t) \\
\left\langle D F_{\varepsilon, n}(t, x), h\right\rangle & =\left(\rho_{\varepsilon} *\left\langle D F\left(\cdot, J_{n} x\right), J_{n} h\right\rangle\right)(t) \\
D^{2} F_{\varepsilon, n}(t, x)(h, k) & =\left(\rho_{\varepsilon} * D^{2} F\left(t, J_{n} x\right)\left(J_{n} h, J_{n} k\right)\right)(t) .
\end{aligned}
$$

Moreover,

$$
\frac{\partial F_{\varepsilon, n}}{\partial t}(t, x)=\left(\rho_{\varepsilon} * \frac{\partial F}{\partial t}\left(\cdot, J_{n} x\right)\right)(t)
$$

on $\mathscr{T} \times D(A)$. To see this take $(t, x) \in \mathscr{T} \times D(A)$, consider the limit

$$
\begin{aligned}
\lim _{a \rightarrow 0} & \frac{1}{a}\left[F_{\varepsilon, n}(t+a, x)-F_{\varepsilon, n}(t, x)\right] \\
\quad & =\lim _{a \rightarrow 0} \frac{1}{a} \int_{\mathbb{R}} \rho_{\varepsilon}(r)\left[F\left(t+a-r, J_{n} x\right)-F\left(t-r, J_{n} x\right)\right] \mathrm{d} r \\
& =\lim _{a \rightarrow 0} \frac{1}{a} \int_{B_{\varepsilon}(0)} \rho_{\varepsilon}(r)\left[F\left(t+a-r, J_{n} x\right)-F\left(t-r, J_{n} x\right)\right] \mathrm{d} r
\end{aligned}
$$

and set $R_{\varepsilon}^{t}:=\left\{r \in B_{\varepsilon}(0): t-r \in \mathscr{T}_{0}\right\}$, where $\mathscr{T}_{0}:=[-1,0) \cup \mathscr{T} \cup(T, T+1]$.

Since $t-R_{\varepsilon}^{t}=\left(t-B_{\varepsilon}(0)\right) \cap \mathscr{T}_{0}$, we have that $\lambda\left(R_{\varepsilon}^{t}\right)=\lambda\left(B_{\varepsilon}(0)\right)$, and hence, we can go on from (6) finding

$$
\begin{aligned}
\lim _{a \rightarrow 0} & \frac{1}{a}\left[F_{\varepsilon, n}(t+a, x)-F_{\varepsilon, n}(t, x)\right] \\
& =\lim _{a \rightarrow 0} \frac{1}{a} \int_{R_{\varepsilon}^{t}} \rho_{\varepsilon}(r)\left[F\left(t+a-r, J_{n} x\right)-F\left(t-r, J_{n} x\right)\right] \mathrm{d} r \\
& =\int_{R_{\varepsilon}^{t}} \rho_{\varepsilon}(r) \frac{\partial F}{\partial t}\left(t-r, J_{n} x\right) \mathrm{d} r \\
& =\left(\rho_{\varepsilon} * \frac{\partial F}{\partial t}\left(\cdot, J_{n} x\right)\right)(t) .
\end{aligned}
$$


Now set $X_{n}(t)=J_{n} X(t), X_{n}^{0}=J_{n} X^{0}, B_{n}(t)=J_{n} B(t), C_{n}(t)=J_{n} C(t)$. Since $J_{n}$ commutes with $e^{t A}$, we have

$$
X_{n}(t)=e^{t A} X_{n}^{0}+\int_{0}^{t} e^{(t-s) A} B_{n}(s) \mathrm{d} s+\int_{0}^{t} e^{(t-s) A} C_{n}(s) \mathrm{d} W(s) .
$$

Moreover, $X_{n}(t), B_{n}(t), C_{n}(t)$ belong to $D(A)$ for a.e. $t \in[0, T]$, with $\left|A X_{n}(\cdot)\right|$ integrable $\mathbb{P}$-a.s.; hence,

$$
X_{n}(t)=X_{n}^{0}+\int_{0}^{t}\left[A X_{n}(s)+B_{n}(s)\right] \mathrm{d} s+\int_{0}^{t} C_{n}(s) \mathrm{d} W(s)
$$

and by the Itô formula in Hilbert spaces given in Proposition 1 we have

$$
\begin{aligned}
F_{\varepsilon, n}\left(t, X_{n}(t)\right)= & F_{\varepsilon, n}\left(0, X_{n}^{0}\right) \\
& +\int_{0}^{t}\left(\left\langle A X_{n}(s), D F_{\varepsilon, n}\left(s, X_{n}(s)\right)\right\rangle+\frac{\partial F_{\varepsilon, n}}{\partial s}\left(s, X_{n}(s)\right)\right) \mathrm{d} s \\
& +\int_{0}^{t}\left\langle B_{n}(s), D F_{\varepsilon, n}\left(s, X_{n}(s)\right)\right\rangle \mathrm{d} s \\
& +\int_{0}^{t}\left\langle D F_{\varepsilon, n}\left(s, X_{n}(s)\right), C_{n}(s) \mathrm{d} W(s)\right\rangle \\
& +\frac{1}{2} \int_{0}^{t} \operatorname{Tr}\left[C_{n}(s) Q C_{n}(s)^{*} D^{2} F_{\varepsilon, n}\left(s, X_{n}(s)\right)\right] \mathrm{d} s
\end{aligned}
$$

Let us prove the convergence (as $n \rightarrow \infty$ and $\varepsilon \rightarrow 0$ ) of each term to the corresponding one of the formula stated by the theorem. We fix $t$ and prove the a.s. (hence in probability) convergence of each term, except for the convergence in probability of the Itô term; this yields the conclusion.

Given $(\omega, t)$, we have $F_{\varepsilon, n}\left(t, X_{n}(\omega, t)\right)=\rho_{\varepsilon} * F\left(\cdot, J_{n}^{2} X(\omega, t)\right)(t)$ and thus

$$
\begin{aligned}
& \left|F_{\varepsilon, n}\left(t, X_{n}(\omega, t)\right)-F(t, X(\omega, t))\right| \\
& \quad=\left|\int_{\mathbb{R}} \rho_{\varepsilon}(r) F\left(t-r, J_{n}^{2} X(\omega, t)\right) \mathrm{d} r-F(t, X(\omega, t))\right| \\
& \quad \leq \int_{B_{\varepsilon}(0)} \rho_{\varepsilon}(r)\left|F\left(t-r, J_{n}^{2} X(\omega, t)\right)-F(t, X(\omega, t))\right| \mathrm{d} r,
\end{aligned}
$$

which is arbitrarily small for $\varepsilon$ small enough and $n$ big enough, because $J_{n}^{2}$ converges strongly to the identity and $F$ is continuous; similarly

$$
\lim _{\substack{\varepsilon \rightarrow 0 \\ n \rightarrow \infty}} F_{\varepsilon, n}\left(0, X_{n}^{0}(\omega)\right)=F\left(0, X^{0}(\omega)\right) .
$$

From now on we work in the set $\Omega_{1}$ where $X$ has relatively compact paths in $\widetilde{E}$ (hence in $H$ ). Fix $\delta>0$. Since for $\omega \in \Omega_{1}$ the set $\{X(\omega, s)\}_{s \in[0, t]}$ is relatively compact, we 
have that $J_{n}^{2} X(s)$ converges uniformly with respect $s$ to $X(s)$, and hence, there exists $N \in \mathbb{N}$ such that for any $n>N\left|J_{n}^{2} X(s)-X(s)\right|<\delta / 2$ for all $s$; moreover, the set $\left\{J_{n} X(s)\right\}_{n, s}$ is bounded.

The family $\left\{B_{\delta / 2}(X(s))\right\}_{s \in[0, t]}$ is an open cover of $\{X(s)\}_{s \in[0, t]}$; by compactness it admits a finite subcover $\left\{B_{\delta / 2}\left(X\left(s_{i}\right)\right)\right\}_{i=1, \ldots, M}$ for some finite set $\left\{s_{1}, \ldots, s_{M}\right\} \subset$ $[0, t]$; therefore, for any $s$ there exists $i \in\{1, \ldots, N\}$ such that $\left|X(s)-X\left(s_{i}\right)\right|<\delta / 2$ and

$$
\left|J_{n}^{2} X(s)-X\left(s_{i}\right)\right| \leq\left|J_{n}^{2} X(s)-X(s)\right|+\left|X(s)-X\left(s_{i}\right)\right|<\delta
$$

for $n>N$ where $N$ does not depend on $s$ since the convergence is uniform. This shows that the set $\left\{J_{n}^{2} X(s)\right\}_{n, s}$ is totally bounded both in $\widetilde{E}$ and in $H$.

Therefore, we can study the convergence of the other terms as follows. First we consider the difference

$$
\begin{aligned}
\left|\int_{0}^{t}\left\langle B_{n}(s), D F_{\varepsilon, n}\left(s, X_{n}(s)\right)\right\rangle \mathrm{d} s-\int_{0}^{t}\langle B(s), D F(s, X(s))\rangle \mathrm{d} s\right| \\
\leq \mid \int_{0}^{t}\left\langle J_{n}^{2} B(s),\left(\rho_{\varepsilon} * D F\left(\cdot, J_{n}^{2} X(s)\right)\right)(s)\right\rangle \mathrm{d} s \\
\quad-\int_{0}^{t}\left\langle J_{n}^{2} B(s), D F(s, X(s))\right\rangle \mathrm{d} s \mid \\
+\left|\int_{0}^{t}\left\langle J_{n}^{2} B(s)-B(s), D F(s, X(s))\right\rangle \mathrm{d} s\right| .
\end{aligned}
$$

The second term in this last sum is bounded by

$$
\int_{0}^{t}\left|J_{n}^{2} B(s)-B(s)\right||D F(s, X(s))| \mathrm{d} s
$$

and $\{X(s)\}_{s}$ is compact, hence $|D F(s, X(s))|$ is bounded uniformly in $s$ and, since the $J_{n}^{2}$ are equibounded and converge strongly to the identity and $B$ is integrable, Lebesgue dominated convergence theorem applies. The first term in the previous sum instead is bounded by

$$
\int_{0}^{t}\left|J_{n}^{2} B(s)\right| \int_{B_{\varepsilon}(0)} \rho_{\varepsilon}(r)\left|D F\left(s-r, J_{n}^{2} X(s)\right)-D F(s, X(s))\right| \mathrm{d} r \mathrm{~d} s
$$

by the discussion above the set $[0, t] \times\left(\left\{J_{n}^{2} X(s)\right\}_{n, s} \cup\{X(s)\}_{s}\right)$ is contained in a compact subset of $[0, T] \times H$, hence $|D F|$ is bounded on that set uniformly in $s$ and $r$. Thanks again to the equicontinuity of the operators $J_{n}^{2}$ and the integrability of $B$, (7) is shown to go to 0 by the dominated convergence theorem and the continuity of $D F$. 
About the critical term involving $G$ we have

$$
\begin{aligned}
\mid \int_{0}^{t}( & \left.\frac{\partial F_{\varepsilon, n}}{\partial t}\left(s, X_{n}(s)\right)+\left\langle A X_{n}(s), D F_{\varepsilon, n}\left(s, X_{n}(s)\right)\right\rangle\right) \mathrm{d} s-\int_{0}^{t} G(s, X(s)) \mathrm{d} s \mid \\
\leq & \int_{[0, t] \cap \mathscr{T}} \mid \rho_{\varepsilon} *\left(\frac{\partial F}{\partial t}\left(\cdot, J_{n}^{2} X(s)\right)+\left\langle A J_{n}^{2} X(s), D F\left(\cdot, J_{n}^{2} X(s)\right)\right\rangle\right)(s) \\
& -G(s, X(s)) \mid \mathrm{d} s \\
= & \int_{[0, t] \cap \mathscr{T}}\left|\left(\rho_{\varepsilon} * G\left(\cdot, J_{n}^{2} X(s)\right)\right)(s)-G(s, X(s))\right| \mathrm{d} s \\
\leq & \int_{[0, t] \cap \mathscr{T}} \int_{B_{\varepsilon}(0)} \rho_{\varepsilon}(r)\left|G\left(s-r, J_{n}^{2} X(s)\right)-G(s, X(s))\right| \mathrm{d} r \mathrm{~d} s
\end{aligned}
$$

and this last quantity goes to 0 by compactness and continuity of $G$ in the same way as the previous term (now with respect to the topology on $\widetilde{E}$ ).

For the Itô term we have

$$
\begin{gathered}
\int_{0}^{t}\left|C^{*}(s)\left(J_{n}^{2}\right)^{*}\left(\rho_{\varepsilon} * D F\left(\cdot, J_{n}^{2} X(s)\right)\right)(s)-C^{*}(s) D F(s, X(s))\right|^{2} \mathrm{~d} s \\
\leq \int_{0}^{t}\|C(s)\|^{2}\left|\left(J_{n}^{*}\right)^{2} \rho_{\varepsilon} * D f\left(\cdot, J_{n}^{2} X(s)\right)(s)-D F(s, X(s))\right|^{2} \mathrm{~d} s
\end{gathered}
$$

writing

$$
\begin{aligned}
& \left|\left(J_{n}^{*}\right)^{2} \rho_{\varepsilon} * D F\left(\cdot, J_{n}^{2} X(s)\right)(s)-D F(s, X(s))\right| \\
& \leq\left|\left(J_{n}^{*}\right)^{2} \rho_{\varepsilon} * D F\left(\cdot, J_{n}^{2} X(s)\right)(s)-\left(J_{n}^{*}\right)^{2} D F(s, X(s))\right| \\
& \quad+\left|\left(J_{n}^{*}\right)^{2} D F(s, X(s))-D F(s, X(s))\right|
\end{aligned}
$$

it is immediate to see that the right-hand side of (8) converges to 0 almost surely, hence

$$
\int_{0}^{t}\left\langle D F_{\varepsilon, n}\left(s, X_{n}(s)\right), C_{n}(s) \mathrm{d} W(s)\right\rangle \rightarrow \int_{0}^{t}\langle D F(s, X(s)), C(s) \mathrm{d} W(s)\rangle
$$

in probability.

It remains to treat the trace term. Let $\left\{h_{j}\right\}$ be an orthonormal complete system in $H$; then

$$
\begin{array}{r}
\mid \int_{0}^{t} \operatorname{Tr}\left[C_{n}(s) Q C_{n}(s)^{*} D^{2} F_{\varepsilon, n}\left(s, X_{n}(s)\right)\right] \mathrm{d} s \\
\quad-\int_{0}^{t} \operatorname{Tr}\left[C(s) Q C(s)^{*} D F(s, X(s))\right] \mathrm{d} s \mid
\end{array}
$$




$$
\begin{aligned}
& \leq \int_{0}^{t} \sum_{j} \mid\left\langle\left[ J_{n} C(s) Q C(s)^{*}\left(J_{n}^{*}\right)^{2} \rho_{\varepsilon} * D^{2} F\left(\cdot, J_{n}^{2} X(s)\right)(s) J_{n}\right.\right. \\
& \left.\left.\quad-C(s) Q C(s)^{*} D^{2} F(s, X(s))\right] h_{j}, h_{j}\right\rangle \mid \mathrm{d} s .
\end{aligned}
$$

Now for any $j$

$$
\begin{aligned}
\mid J_{n} C(s) Q C(s)^{*}\left(J_{n}^{*}\right)^{2} \rho_{\varepsilon} * D^{2} F\left(\cdot, J_{n}^{2} X(s)\right)(s) J_{n} h_{j} \\
\quad-C(s) Q C(s)^{*} D^{2} F(s, X(s)) h_{j} \mid \\
\leq \mid J_{n} C(s) Q C(s)^{*}\left(J_{n}^{*}\right)^{2} \rho_{\varepsilon} * D^{2} F\left(\cdot, J_{n}^{2} X(s)\right)(s) J_{n} h_{j} \\
\quad-C(s) Q C(s)^{*} D^{2} F(s, X(s)) J_{n} h_{j} \mid \\
\quad+\left\|C(s) Q C(s)^{*} D^{2} F(s, X(s))\right\| \cdot\left|J_{n} h_{j}-h_{j}\right| .
\end{aligned}
$$

The second term in the sum converges to 0 thanks to the properties of $J_{n}$; the first one is bounded by the sum

$$
\begin{aligned}
& \mid J_{n} C(s) Q C(s)^{*}\left(J_{n}^{*}\right)^{2} \rho_{\varepsilon} * D^{2} F\left(\cdot, J_{n}^{2} X(s)\right)(s) J_{n} h_{j} \\
& \quad-J_{n} C(s) Q C(s)^{*}\left(J_{n}^{*}\right)^{2} D^{2} F(s, X(s)) J_{n} h_{j} \mid \\
& \quad+\left|\left[J_{n} C(s) Q C(s)^{*}\left(J_{n}^{*}\right)^{2}-C(s) Q C(s)^{*}\right] D^{2} F(s, X(s)) J_{n} h_{j}\right|,
\end{aligned}
$$

whose first addend is less or equal to $\left\|J_{n} C(s) Q C(s)^{*}\left(J_{n}^{*}\right)^{2}\right\| \int_{B_{\varepsilon}(0)} \rho_{\varepsilon}\left|D^{2} F\left(s-r, J_{n}^{2} X(s)\right)-D^{2} F(s, X(s))\right|\left|J_{n} h_{j}\right| \mathrm{d} r$

which is shown to go to zero as before. For the second addend of (10) notice that for any $k \in H$

$$
\begin{aligned}
& \left|\left[J_{n} C(s) Q C(s)^{*}\left(J_{n}^{*}\right)^{2}-C(s) Q C(s)^{*}\right] k\right| \\
& \leq\left|\left[J_{n} C(s) Q C(s)^{*}\left(J_{n}^{*}\right)^{2}-J_{n} C(s) Q C(s)^{*}\right] k\right| \\
& \quad+\left|\left[J_{n} C(s) Q C(s)^{*}-C(s) Q C(s)^{*}\right] k\right| \\
& \leq|| J_{n} C(s) Q C(s)^{*} \|\left|\left(J_{n}^{*}\right)^{2} k-k\right| \\
& \quad+\left|J_{n} C(s) Q C(s)^{*} k-C(s) Q C(s)^{*} k\right|
\end{aligned}
$$

which tends to 0 as $n$ tends to $\infty$.

The same compactness arguments used in the previous steps, the continuity of $D^{2} F$ and the equiboundedness of the family $\left\{J_{n}\right\}$ allow to apply Lebesgue dominated 
convergence theorem both to the series and to the integral with respect to $s$ in (9). This concludes the proof.

\section{Extension to Particular Banach Spaces}

In this section we consider the following framework. Let $H_{1}$ be a separable Hilbert space with scalar product $\langle\cdot\rangle_{1}$ and norm $\|\cdot\|_{1}$ and let $E_{2}$ be a Banach space, with norm $\|\cdot\|_{E_{2}}$ and duality pairing denoted by $\langle\cdot, \cdot\rangle$, densely and continuously embedded in another separable Hilbert space $\mathrm{H}_{2}$ with scalar product and norm denoted, respectively, by $\langle\cdot\rangle_{2}$ and $\|\cdot\|_{2}$. Then set $H:=H_{1} \times H_{2}$ so that

$$
E:=H_{1} \times E_{2} \subset H
$$

with continuous and dense embedding. We adopt here the standard identification of $H$ with $H^{*}$ so that

$$
E \subset H \cong H^{*} \subset E^{*} .
$$

Our aim here is to extend the results exposed so far to situations in which the process $X$ lives in a subset of $E$ but the noise only acts on $H_{1}$.

Example 1 In the application of this abstract framework to path-dependent functionals (see Sect. 5), we will choose the spaces

$$
H=\mathbb{R}^{d} \times L^{2}\left(-T, 0 ; \mathbb{R}^{d}\right)
$$

and

$$
E=\mathbb{R}^{d} \times\left\{\varphi \in C\left([-T, 0) ; \mathbb{R}^{d}\right): \exists \lim _{s \rightarrow 0^{-}} \varphi(s) \in \mathbb{R}^{d}\right\} .
$$

Similarly to the setup we introduced in Sect. 2, consider a complete probability space $(\Omega, \mathscr{F}, \mathbb{P})$ with a complete filtration $\mathbb{F}=\left(\mathscr{F}_{t}\right)_{t \geq 0}$ and a Wiener process $(W(t))_{t \geq 0}$ in another separable Hilbert space $U$ with nuclear covariance operator $Q$.

Consider a linear operator $A$ on $H$ with domain $D(A) \subset E$ and assume that it generates a strongly continuous semigroup $e^{t A}$ in $H$. Let $B: \Omega \times[0, T] \rightarrow E$ be a progressively measurable process s.t. $\int_{0}^{t}|B(t)| \mathrm{d} t<\infty$ as in Sect. 2; let then $\widetilde{C}: \Omega \times[0, T] \rightarrow L\left(U, H_{1}\right)$ be another progressively measurable process that satisfies $\int_{0}^{T}\|C(t)\|_{L\left(U, H_{1}\right)}^{2} \mathrm{~d} t<\infty$ and define $C: \Omega \times[0, T] \rightarrow L(U, E)$ as

$$
C(t) u=\left(\begin{array}{c}
\widetilde{C}(t) u \\
0
\end{array}\right), \quad u \in U
$$

let $X^{0}$ be a $\mathscr{F} 0$-measurable random vector with values in $H$ and set

$$
X(t)=e^{t A} X^{0}+\int_{0}^{t} e^{(t-s) A} B(s) \mathrm{d} s+\int_{0}^{t} e^{(t-s) A} C(s) \mathrm{d} W(s)
$$


Finally set

$$
\widetilde{E}=\overline{D(A)}^{E}, \quad \widetilde{D}=A^{-1}(E) .
$$

Notice that $\widetilde{D} \subset D(A) \subset \widetilde{E}$. In most examples the set $\widetilde{D}$ is not dense in $E$. As in Sect. 2 we assume here that $e^{t A}$ is strongly continuous in $\widetilde{E}$ (and this in turn implies that $\widetilde{D}$ is dense in $\widetilde{E}), X(t)$ actually belongs to $\widetilde{E}$ and that almost surely the set $\{X(t)\}_{t \in[0, T]}$ is relatively compact in $E$.

Example 2 In the path-dependent case, we will have

$$
\begin{aligned}
D(A) & =\left\{\left(\begin{array}{l}
x_{1} \\
x_{2}
\end{array}\right) \in H: x_{2} \in W^{1,2}\left(-T, 0 ; \mathbb{R}^{d}\right), x_{1}=\lim _{s \rightarrow 0^{-}} x_{2}(s)\right\}, \\
A & =\left(\begin{array}{ll}
0 & 0 \\
0 & \frac{d}{d r}
\end{array}\right), \\
\widetilde{E} & =\left\{\left(\begin{array}{l}
x_{1} \\
x_{2}
\end{array}\right) \in E: x_{1}=\lim _{s \rightarrow 0^{-}} x_{2}(s)\right\}, \\
\widetilde{D} & =\left\{\left(\begin{array}{l}
x_{1} \\
x_{2}
\end{array}\right) \in E: x_{2} \in C^{1}\left([-T, 0) ; \mathbb{R}^{d}\right), x_{1}=\lim _{s \rightarrow 0^{-}} x_{2}(s)\right\} .
\end{aligned}
$$

Finally consider a sequence $\mathscr{J}_{n}$ of linear continuous operators, $\mathscr{J}_{n}: H \rightarrow E$ with the properties:

(i) $\mathscr{J}_{n} x \in \widetilde{D}$ for every $x \in \widetilde{E}$;

(ii) $\mathscr{J}_{n} x \rightarrow x$ in the topology of $E$ for every $x \in E$;

(iii) $\mathscr{J}_{n}$ commutes with $A$ on $D(A)$.

By Banach-Steinhaus and Ascoli-Arzelà theorems it follows that the operators $\mathscr{J}_{n}$ are equibounded and converge to the identity uniformly on compact sets of $E$.

Theorem 2 Assume there exists a sequence $\mathscr{J}_{n}$ as above and let $F \in C([0, T] \times E ; \mathbb{R})$ be twice differentiable with respect to its second variable with $D F \in C\left([0, T] \times E ; E^{*}\right)$ and $D^{2} F \in C\left([0, T] \times E ; L\left(E ; E^{*}\right)\right)$. Assume the time derivative $\frac{\partial F}{\partial t}(t, x)$ exists for $(t, x) \in \mathscr{T} \times \widetilde{D}$ where $\mathscr{T} \subset[0, T]$ has Lebesgue measure $\lambda(\mathscr{T})=T$ and does not depend on $x$. If there exists a continuous function $G:[0, T] \times \widetilde{E} \rightarrow \mathbb{R}$ such that

$$
G(t, x)=\frac{\partial F}{\partial t}(t, x)+\langle A x, D F(t, x)\rangle \quad \forall x \in \widetilde{D}, \forall t \in \mathscr{T},
$$

then, in probability,

$$
\begin{aligned}
F(t, X(t))= & F\left(0, X^{0}\right)+\int_{0}^{t} G(s, X(s)) \mathrm{d} s \\
& +\int_{0}^{t}\left(\langle B(s), D F(s, X(s))\rangle+\frac{1}{2} \operatorname{Tr}_{H_{1}}\left[C(s) Q C(s)^{*} D^{2} F(s, X(s))\right]\right) \mathrm{d} s \\
& +\int_{0}^{t}\langle D F(s, X(s)), C(s) \mathrm{d} W(s)\rangle,
\end{aligned}
$$


where $\operatorname{Tr}_{H_{1}}$ is defined for $T \in L(E, E)$ as

$$
\operatorname{Tr}_{H_{1}} T=\sum_{j}\left\langle T\left(\begin{array}{c}
h_{j} \\
0
\end{array}\right),\left(\begin{array}{c}
h_{j} \\
0
\end{array}\right)\right\rangle,
$$

$\left\{h_{j}\right\}$ being an orthonormal complete system in $H_{1}$.

Proof Set $F_{n}:[0, T] \times H \rightarrow \mathbb{R}, F_{n}(t, x):=F\left(t, \mathscr{J}_{n} x\right)$. Thanks to the assumptions on $F$ we have that $F_{n}$ is twice differentiable with respect to the variable $x$ and

$$
\begin{aligned}
D F_{n}(t, x) & =\mathscr{J}_{n}^{*} D F\left(t, \mathscr{J}_{n} x\right) \in L(H ; \mathbb{R}) \cong H \\
D^{2} F_{n}(t, x) & =\mathscr{J}_{n}^{*} D^{2} F\left(t, \mathscr{J}_{n} x\right) \mathscr{J}_{n} \in L(H ; H) .
\end{aligned}
$$

Furthermore for any $t \in \mathscr{T}$ the derivative of $F_{n}$ with respect to $t$ is defined for all $x \in H$ and equals

$$
\frac{\partial F_{n}}{\partial t}(t, x)=\frac{\partial F}{\partial t}\left(t, \mathscr{J}_{n} x\right) .
$$

Set $G_{n}:[0, T] \times \widetilde{E} \rightarrow \mathbb{R}, G_{n}(t, x):=G\left(t, \mathscr{J}_{n} x\right) . G_{n}$ is obviously continuous; we check now that for any $t \in \mathscr{T} G_{n}(t, \cdot)$ extends $\frac{\partial F_{n}}{\partial t}(t, \cdot)+\left\langle A \cdot, D F_{n}(t, \cdot)\right\rangle$ from $D(A)$ to $\widetilde{E}$. Since $\mathscr{J}_{n}$ maps $\widetilde{E}$ into $\widetilde{D} \subset D(A) \subset H$ we have

$$
\begin{aligned}
G_{n}(t, x) & =G\left(t, \mathscr{J}_{n} x\right) \\
& =\frac{\partial F}{\partial t}\left(t, \mathscr{J}_{n} x\right)+\left\langle A \mathscr{J}_{n} x, D F\left(t, \mathscr{J}_{n} x\right)\right\rangle
\end{aligned}
$$

if we choose $x \in D(A), \mathscr{J}_{n}$ commutes with $A$ so that we can proceed to get

$$
\begin{aligned}
& =\frac{\partial F}{\partial t}\left(t, \mathscr{J}_{n} x\right)+\left\langle\mathscr{J}_{n} A x, D F\left(t, \mathscr{J}_{n} x\right)\right\rangle \\
& =\frac{\partial F_{n}}{\partial t}(t, x)+\left\langle A x, D F_{n}(t, x)\right\rangle .
\end{aligned}
$$

Notice that here only the term $\left\langle A x, D F_{n}(t, x)\right\rangle$ has to be extended (since it is not well defined outside $D(A)$ ) while the time derivative of $F_{n}$ makes sense on the whole space $H$ by definition.

We can now apply Theorem 1 to $F_{n}$ and $G_{n}$, obtaining that for each $n$

$$
\begin{aligned}
F_{n}(t, X(t))= & F_{n}\left(0, X^{0}\right)+\int_{0}^{t} G_{n}(s, X(s)) \mathrm{d} s \\
& +\int_{0}^{t}\left[\left\langle B(s), D F_{n}(s, X(s))\right\rangle+\frac{1}{2} \operatorname{Tr}\left[C(s) Q C(s)^{*} D^{2} F_{n}(s, X(s))\right]\right] \mathrm{d} s \\
& +\int_{0}^{t}\left\langle D F_{n}(s, X(s)), C(s) \mathrm{d} W(s)\right\rangle .
\end{aligned}
$$


Here $C(s) Q C(s)^{*}$ maps $E^{*}$ into $E$, therefore $C(s) Q C(s)^{*} D^{2} F_{n}(s, X(s))$ maps $H$ into $E \subset H$ and the trace term can be interpreted as in $H$. Also, since $C(s)$ belongs to $L\left(U ; H_{1} \times\{0\}\right)$, we have that the stochastic integral above is well defined as a stochastic integral in a Hilbert space.

Substituting the definition of $F_{n}$ and identities (13), (14) in the previous equation we get

$$
\begin{aligned}
F & \left(t, \mathscr{J}_{n} X(t)\right)=F\left(0, \mathscr{J}_{n} X^{0}\right)+\int_{0}^{t} G\left(s, \mathscr{J}_{n} X(s)\right) \mathrm{d} s \\
& +\int_{0}^{t}\left[\left\langle\mathscr{J}_{n} B(s), D F\left(s, \mathscr{J}_{n} X(s)\right)\right\rangle+\frac{1}{2} \operatorname{Tr}\left[C(s) Q C(s)^{*} \mathscr{J}_{n}^{*} D^{2} F\left(s, \mathscr{J}_{n} X(s)\right) \mathscr{J}_{n}\right]\right] \mathrm{d} s \\
& +\int_{0}^{t}\left\langle D F\left(s, \mathscr{J}_{n} X(s)\right), \mathscr{J}_{n} C(s) \mathrm{d} W(s)\right\rangle .
\end{aligned}
$$

Now we fix $(\omega, t)$ and study the convergence of each of the terms above. Since $X(\omega, t) \in \widetilde{E}, \mathscr{J}_{n} X(\omega, t) \rightarrow X(\omega, t)$ almost surely as $n \rightarrow \infty$ and therefore by continuity of $F$ we have that $F\left(t, \mathscr{J}_{n} X(\omega, t)\right)$ converges to $F(t, X(\omega, t))$ almost surely. For the same reasons $F\left(0, \mathscr{J}_{n} X^{0}(\omega)\right)$ converges to $F\left(0, X^{0}(\omega)\right)$ almost surely.

Denote by $\Omega_{1}$ the set of full probability where each of the trajectories $\{X(\omega, t)\}_{t}$ is relatively compact. Arguing as in Proof of Theorem 1 it can be shown that, thanks to the uniform convergence on compact sets of the $\mathscr{J}_{n}$, the set $\left\{\mathscr{J}_{n} X(\omega, t)\right\}_{n, t}$ is totally bounded in $E$ for any $\omega \in \Omega_{1}$. Therefore, the a.s. convergence of the terms $\int_{0}^{t} G\left(s, \mathscr{J}_{n} X(\omega, s)\right) \mathrm{d} s$ and $\int_{0}^{t}\left\langle\mathscr{J}_{n} B(\omega, s), D F\left(s, \mathscr{J}_{n} X(\omega, s)\right)\right\rangle \mathrm{d} s$ follows from the dominated convergence theorem since $G$ and $D F$ are continuous, $B$ is integrable, and the family $\left\{\mathscr{J}_{n}\right\}$ is equibounded.

To show the convergence of the stochastic integral term consider

$$
\begin{aligned}
& \int_{0}^{t}\left\|C(s)^{*} \mathscr{J}_{n}^{*} D F\left(s, \mathscr{J}_{n} X(s)\right)-C(s)^{*} D F(s, X(s))\right\|_{U}^{2} \mathrm{~d} s \\
& \quad \leq \int_{0}^{t}\|C(s)\|_{L(U, E)}^{2}\left\|\mathscr{J}_{n}^{*} D F\left(s, \mathscr{J}_{n} X(s)\right)-D F(s, X(s))\right\|_{E^{*}}^{2} \mathrm{~d} s .
\end{aligned}
$$

Now

$$
\begin{aligned}
\left\|\mathscr{J}_{n}^{*} D F\left(s, \mathscr{J}_{n} X(s)\right)-D F(s, X(s))\right\|_{E^{*}} \\
=\sup _{\substack{e \in E \\
\|e\|=1}}\left|\left\langle e, \mathscr{J}_{n}^{*} D F\left(s, \mathscr{J}_{n} X(s)\right)-D F(s, X(s))\right\rangle\right| \\
=\sup _{\substack{e \in E \\
\|e\|=1}}\left|\left\langle\mathscr{J}_{n} e, D F\left(s, \mathscr{J}_{n} X(s)\right)\right\rangle-\langle e, D F(s, X(s))\rangle\right| \\
\leq \sup _{\substack{e \in E \\
\|e\|=1}}\left[\left|\left\langle\mathscr{J}_{n} e, D F\left(s, \mathscr{J}_{n} X(s)\right)\right\rangle-\left\langle\mathscr{J}_{n} e, D F(s, X(s))\right\rangle\right|\right. \\
\left.\quad+\left|\left\langle\mathscr{J}_{n} e-e, D F(s, X(s))\right\rangle\right|\right]
\end{aligned}
$$




$$
\begin{aligned}
& \leq \sup _{\substack{e \in E \\
\|e\|=1}}\left[\left\|\mathscr{J}_{n}\right\|_{E}\left\|D F\left(s, \mathscr{J}_{n} X(s)\right)-D F(s, X(s))\right\|_{E^{*}}\right. \\
& \left.\quad+\left\|\mathscr{J}_{n} e-e\right\|_{E}\|D F(s, X(s))\|_{E^{*}}\right]
\end{aligned}
$$

and this last quantity converges to zero as before, since $\left\{\mathscr{J}_{n}\right\}$ is equibounded, $D F$ is continuous (hence uniformly continuous on $\left.\left\{\mathscr{J}_{n} X(s)\right\}_{n, s} \cup\{X(s)\}_{s}\right)$, and $\mathscr{J}_{n}$ converges to the identity on $E$. Since $\|C(s)\|^{2}$ is integrable, we can apply again the dominated convergence theorem in (16) to get that the left-hand side converges to 0 almost surely, hence

$$
\int_{0}^{t}\left\langle D F\left(s, \mathscr{J}_{n} X(s)\right), \mathscr{J}_{n} C(s) \mathrm{d} W(s)\right\rangle \rightarrow \int_{0}^{t}\langle D F(s, X(s)), C(s) \mathrm{d} W(s)\rangle
$$

in probability.

It remains to study the trace term. First notice that, since $E^{*}=\left(H_{1} \times E_{2}\right)^{*} \cong$ $H_{1}^{*} \times E_{2}^{*} \cong H_{1} \times E_{2}^{*}$, every $f \in E^{*}$ can be written as a couple $\left(f_{1}, f_{2}\right) \in H_{1} \times E_{2}^{*}$ and therefore for any $u \in U$ and $f \in E^{*}$

$$
\begin{aligned}
{ }_{E}\langle C(s) u, f\rangle_{E^{*}} & ={ }_{E}\left\langle\left(\begin{array}{c}
\widetilde{C}(s) u \\
0
\end{array}\right),\left(\begin{array}{c}
f_{1} \\
f_{2}
\end{array}\right)\right\rangle_{E^{*}} \\
& =\left\langle\widetilde{C}(s) u, f_{1}\right\rangle_{1}={ }_{U}\left\langle u, \widetilde{C}(s)^{*} f_{1}\right\rangle_{U} ;
\end{aligned}
$$

hence $C(s)^{*} f=\widetilde{C}(s)^{*} f_{1}$ for any $f \in E^{*}$.

Now let $\mathscr{H}_{1}$ and $\mathscr{H}_{2}$ be complete orthonormal systems of $H_{1}$ and $H_{2}$, respectively, and set $\mathscr{H}_{1}:=\mathscr{H}_{1} \times\{0\}, \mathscr{H}_{2}:=\mathscr{H}_{2} \times\{0\}$, so that $\mathscr{H}:=\mathscr{H}_{1} \cup \mathscr{H}_{2}$ is a complete orthonormal system for $H$. $\mathscr{H}$ is countable since $H_{1}$ and $H_{2}$ are separable. For $h \in \mathscr{H}$ we have that

$$
y:=\mathscr{J}_{n}^{*} D^{2} F\left(s, \mathscr{J}_{n} X(s)\right) \mathscr{J}_{n} h \in H \subset E^{*}=H_{1} \times E_{2}^{*},
$$

so that, writing $y=\left(y_{1}, y_{2}\right)$, we have

$$
C(s) Q C(s)^{*} y=C(s) Q \widetilde{C}(s)^{*} y_{1}=\left(\begin{array}{c}
\widetilde{C}(s) Q \widetilde{C}(s)^{*} y_{1} \\
0
\end{array}\right) \in H_{1} \times\{0\} \subset E \subset H .
$$

Therefore,

$$
\left\langle C(s) Q C(s)^{*} \mathscr{J}_{n}^{*} D^{2} F\left(s, \mathscr{J}_{n} X(s)\right) \mathscr{J}_{n} h, h\right\rangle=\left\langle\left(\begin{array}{c}
\widetilde{C}(s) Q \widetilde{C}(s)^{*} y_{1} \\
0
\end{array}\right), h\right\rangle
$$

and this last quantity can be different from 0 only if $h \in \mathscr{H}_{1}$. This implies

$$
\begin{aligned}
\operatorname{Tr} & {\left[C(s) Q C(s)^{*} \mathscr{J}_{n}^{*} D^{2} F\left(s, \mathscr{J}_{n} X(s)\right) \mathscr{J}_{n}\right] } \\
& =\sum_{h \in \mathscr{H}}\left\langle C(s) Q C(s)^{*} \mathscr{J}_{n}^{*} D^{2} F\left(s, \mathscr{J}_{n} X(s)\right) \mathscr{J}_{n} h, h\right\rangle
\end{aligned}
$$




$$
\begin{aligned}
& =\sum_{h \in \mathscr{H}_{1}}\left\langle C(s) Q C(s)^{*} \mathscr{J}_{n}^{*} D^{2} F\left(s, \mathscr{J}_{n} X(s)\right) \mathscr{J}_{n} h, h\right\rangle_{1} \\
& =\operatorname{Tr}_{H_{1}}\left[C(s) Q C(s)^{*} \mathscr{J}_{n}^{*} D^{2} F\left(s, \mathscr{J}_{n} X(s)\right) \mathscr{J}_{n}\right] .
\end{aligned}
$$

Now, setting $\widetilde{K}:=\sup _{n}\left\|\mathscr{J}_{n}\right\|$ we have that for $h \in \mathscr{H}_{1}$

$$
\begin{aligned}
\mid \operatorname{Tr}_{H_{1}}[ & \left.C(s) Q C(s)^{*} \mathscr{J}_{n}^{*} D^{2} F\left(s, \mathscr{J}_{n} X(s)\right) \mathscr{J}_{n}\right]-\operatorname{Tr}_{H_{1}}\left[C(s) Q C(s)^{*} D^{2} F(s, X(s))\right] \mid \\
= & \mid \sum_{h \in \mathscr{H}_{1}}\left\langle D^{2} f\left(t, \mathscr{J}_{n} X(s)\right) \mathscr{J}_{n} h, \mathscr{J}_{n} C(s) Q C(s)^{*} h\right\rangle \\
& -\sum_{h \in \mathscr{H}_{1}}\left\langle D^{2} F(t, X(s)) h, C(s) Q C(s)^{*} h\right\rangle \mid \\
\leq & \sum_{h \in \mathscr{H}_{1}}\left|\left\langle D^{2} F\left(t, \mathscr{J}_{n} X(s)\right) \mathscr{J}_{n} h, \mathscr{J}_{n} C(s) Q C(s)^{*} h-C(s) Q C(s)^{*} h\right\rangle\right| \\
& +\sum_{h \in \mathscr{H}_{1}}\left|\left\langle D^{2} F\left(t, \mathscr{J}_{n} X(s)\right) \mathscr{J}_{n} h-D^{2} F(t, X(s)) h, C(s) Q C(s)^{*} h\right\rangle\right| \\
\leq & \widetilde{K}\left\|D^{2} F\left(t, \mathscr{J}_{n} X(s)\right)\right\| \sum_{h \in \mathscr{H}_{1}}\left|\mathscr{J}_{n} C(s) Q C(s)^{*} h-C(s) Q C(s)^{*} h\right| \\
& +\|C(s)\|_{L(U, E)}^{2}\|Q\|_{L(U, U)}^{2} \sum_{h \in \mathscr{H}_{1}}\left[\widetilde{K}\left\|D^{2} F\left(t, \mathscr{J}_{n} X(s)\right)-D^{2} F(t, X(s))\right\|\right. \\
& \left.+\left\|D^{2} F(t, X(s))\right\|\left|\mathscr{J}_{n} h-h^{2}\right|\right] ;
\end{aligned}
$$

therefore thanks to the equiboundedness of $\left\{\mathscr{J}_{n}\right\}$ and the uniform continuity of $D^{2} F$ on the set $\left\{\mathscr{J}_{n} X(s)\right\}_{n, s} \cup\{X(s)\}_{s}$ we can apply the dominated convergence theorem to the sum over $h \in \mathscr{H}_{1}$ to obtain that

$$
\begin{aligned}
& \operatorname{Tr}_{H_{1}}\left[C(s) Q C(s)^{*} \mathscr{J}_{n}^{*} D^{2} F\left(s, \mathscr{J}_{n} X(s)\right) \mathscr{J}_{n}\right] \\
& \stackrel{n \rightarrow \infty}{\longrightarrow} \operatorname{Tr}_{H_{1}}\left[C(s) Q C(s)^{*} D^{2} F(s, X(s))\right] .
\end{aligned}
$$

Since $D^{2} F$ is bounded also in $s \in[0, T]$ and $\|C(s)\|_{L(U ; E)}^{2}$ is integrable by assumption, a second application of the dominated convergence theorem yields that for every $t \in[0, T]$

$$
\begin{aligned}
& \int_{0}^{t} \operatorname{Tr}_{H_{1}}\left[C(s) Q C(s)^{*} \mathscr{J}_{n}^{*} D^{2} F\left(s, \mathscr{J}_{n} X(s)\right) \mathscr{J}_{n}\right] \mathrm{d} s \\
& \stackrel{n \rightarrow \infty}{\longrightarrow} \int_{0}^{t} \operatorname{Tr}_{H_{1}}\left[C(s) Q C(s)^{*} D^{2} F(s, X(s))\right] \mathrm{d} s,
\end{aligned}
$$

thus concluding the proof. 
Remark 1 The use of both spaces $E$ and $\widetilde{E}$ in the statement of the theorem can seem unjustified at first sight: since the process $X$ is supposed to live in $\widetilde{E}$ and the result is a Itô formula valid on $\widetilde{E}$ (because the extension $G$ is defined on $\widetilde{E}$ only), everything could apparently be formulated in $\widetilde{E}$. However, in most examples the space $\widetilde{E}$ is not a product space (see Sect. 5) hence neither is its dual space, and the product structure of the dual is needed to show that the second order term is concentrated only on the $H_{1}$-component. Since asking $F$ to be defined on $[0, T] \times H$ will leave out many interesting examples (we typically want to endow $\widetilde{E}$ with a topology stronger that the one of $H$ ), the choice to use the intermediate space $E$ seems to be the more adequate.

Corollary 1 Consider $n+1$ points $0=t_{0} \leq t_{1} \leq \cdots \leq t_{n}=T$ and assume that $F \in C\left(\left[t_{j}, t_{j+1}\right) \times E ; \mathbb{R}\right)$ for $j=0,1, \ldots, n-1$. Suppose, moreover, that

1. the map $y \mapsto F(t, y)$ is twice differentiable for every $t \in[0, T]$;

2. $D F \in C\left(\left[t_{j}, t_{j+1}\right) \times E ; E^{*}\right)$ for $j=0,1, \ldots, n-1$;

3. $D^{2} F \in C\left(\left[t_{j}, t_{j+1}\right) \times E ; L\left(E, E^{*}\right)\right)$ for $j=0,1, \ldots, n-1$;

4. the map $t \mapsto F(t, y)$ is càdlàg for every $y \in E$;

5. $\frac{\partial F}{\partial t}$ exists for $(t, x) \in \mathscr{T} \times \widetilde{D}$ where $\mathscr{T}$ is as in Theorem 2 ;

6. there exists a function $G$ such that $G \in C\left(\left[t_{j}, t_{j+1}\right) \times \widetilde{E} ; \mathbb{R}\right)$ for all $j$ and such that

$$
G(t, x)=\frac{\partial F}{\partial t}(t, x)+\langle A x, D F(t, x)\rangle \quad \forall x \in \widetilde{D}, \forall t \in \mathscr{T} \cap\left[t_{j}, t_{j+1}\right) .
$$

Then the formula

$$
\begin{aligned}
F(T, X(T))= & F\left(0, X^{0}\right)+\sum_{j=1}^{n}\left[F\left(t_{j}, X\left(t_{j}\right)\right)-F\left(t_{j}-, X\left(t_{j}\right)\right)\right] \\
& +\int_{0}^{T} G(s, X(s)) \mathrm{d} s+\int_{0}^{T}\langle D F(s, X(s)), C(s) \mathrm{d} W(s)\rangle \\
& +\int_{0}^{T}\left(\langle B(s), D F(s, X(s))\rangle+\frac{1}{2} \operatorname{Tr}_{H_{1}}\left[C(s) Q C(s)^{*} D^{2} F(s, X(s))\right]\right) \mathrm{d} s
\end{aligned}
$$

holds.

Proof Thanks to the assumptions, Theorem 2 can be applied to obtain $n$ identities for the increments $F\left(t_{j+1}-\varepsilon, X\left(t_{j+1}-\varepsilon\right)\right)-F\left(t_{j}, X\left(t_{j}\right)\right), j=0, \ldots, n-1$, with $0<\varepsilon<\min _{j}\left(t_{j+1}-t_{j}\right)$. Summing up these identities and taking the limit as $\varepsilon$ goes to 0 yield the result.

\section{Application to Generators of Groups}

In a Hilbert space $H$, given a Wiener process $(W(t))_{t \geq 0}$ with covariance $Q$, defined on a filtered probability space $\left(\Omega, \mathscr{F},\left(\mathscr{F}_{t}\right)_{t \geq 0}, P\right)$, given $x^{0} \in H, B: \Omega \times[0, T] \rightarrow H$ progressively measurable and integrable in $t, P$-a.s., $C: \Omega \times[0, T] \rightarrow L(H, H)$ 
progressively measurable and square integrable in $t, P$-a.s., let $X(t)$ be the stochastic process given by the mild formula

$$
X(t)=e^{t A} x^{0}+\int_{0}^{t} e^{(t-s) A} B(s) \mathrm{d} s+\int_{0}^{t} e^{(t-s) A} C(s) \mathrm{d} W(s),
$$

where $e^{t A}$ is a strongly continuous group. In this particular case we can also write

$$
X(t)=e^{t A}\left(x^{0}+\int_{0}^{t} e^{-s A} B(s) \mathrm{d} s+\int_{0}^{t} e^{-s A} C(s) \mathrm{d} W(s)\right),
$$

from which we may deduce, for instance, that $X$ is a continuous process in $H$. Formally

$$
\mathrm{d} X(t)=A X(t) \mathrm{d} t+B(t) \mathrm{d} t+C(t) \mathrm{d} W(t)
$$

but $A X(t)$ is generally not well defined: typically the solution has the same spatial regularity of the initial condition and the forcing terms. Thus in general, one cannot apply the classical Itô formula to $F(t, X(t))$, due to this fact. A possibility is given by the mild Itô formula [6]. We show here an alternative, which applies when suitable cancellations in $F(t, x)$ occur.

As a first example, let $F(t, x)$ be given by

$$
F(t, x)=F_{0}\left(e^{-t A} x\right)+\int_{0}^{t} H_{0}\left(s, e^{-(t-s) A} x\right) \mathrm{d} s
$$

where $F_{0} \in C^{2}(H ; \mathbb{R}), H_{0} \in C([0, T] \times H ; \mathbb{R})$, with continuous derivatives $D H_{0}$, $D^{2} H_{0}$. Then $\frac{\partial F}{\partial t}(t, x)$ exists for all $x \in D(A), t \in[0, T]$ and it is given by

$$
\begin{aligned}
\frac{\partial F}{\partial t}(t, x)= & -\left\langle\left(D F_{0}\right)\left(e^{-t A} x\right), e^{-t A} A x\right\rangle+H_{0}(t, x) \\
& -\int_{0}^{t}\left\langle\left(D H_{0}\right)\left(s, e^{-(t-s) A} x\right), e^{-(t-s) A} A x\right\rangle \mathrm{d} s .
\end{aligned}
$$

Moreover, $D F \in C([0, T] \times H ; H), D^{2} F \in C([0, T] \times H ; L(H, H))$ and

$$
\begin{aligned}
\langle D F(t, x), h\rangle= & \left\langle\left(D F_{0}\right)\left(e^{-t A} x\right), e^{-t A} h\right\rangle \\
& +\int_{0}^{t}\left\langle D H_{0}\left(s, e^{-(t-s) A} x\right), e^{-(t-s) A} h\right\rangle \mathrm{d} s .
\end{aligned}
$$

Therefore,

$$
\frac{\partial F}{\partial t}(t, x)+\langle A x, D F(t, x)\rangle=H_{0}(t, x) .
$$


Consider the function $G(t, x):=\frac{\partial F}{\partial t}(t, x)+\langle A x, D F(t, x)\rangle$. It is a priori well defined only on $x \in D(A)$. However, being

$$
G(t, x)=H_{0}(t, x),
$$

the function $G$ extends to a continuous function on $[0, T] \times H$. Then Theorem 1 applies and Itô formula reads

$$
\begin{aligned}
& F(t, X(t))=F\left(0, x^{0}\right)+\int_{0}^{t} H_{0}(s, X(s)) \mathrm{d} s+\int_{0}^{t}\langle B(s), D F(s, X(s))\rangle \mathrm{d} s \\
& \quad+\int_{0}^{t}\langle D F(s, X(s)), C(s) \mathrm{d} W(s)\rangle+\frac{1}{2} \int_{0}^{t} \operatorname{Tr}\left(C(s) Q C^{*}(s) D^{2} F(s, X(s))\right) \mathrm{d} s .
\end{aligned}
$$

\subsection{Kolmogorov Equation for SDEs with Group Generator}

The previous example concerns a very particular class of functionals $F$. As a more useful (but very related) example, assume we have a solution $F(t, x)$ of the following Kolmogorov equation

$$
\begin{aligned}
& \frac{\partial F}{\partial t}(t, x)+\langle A x+B(t, x), D F(t, x)\rangle \\
& \quad+\frac{1}{2} \operatorname{Tr}\left(C(t, x) Q C^{*}(t, x) D^{2} F(t, X(t))\right)=0,
\end{aligned}
$$

for $x \in D(A), t \in[0, T], F(T, x)=\varphi(x)$, with the regularity

$$
\begin{aligned}
F & \in C([0, T] \times H ; \mathbb{R}), \quad D F \in C([0, T] \times H ; H) \\
D^{2} F & \in C([0, T] \times H ; L(H, H)), \quad \frac{\partial F}{\partial t} \in C([0, T] \times D(A) ; \mathbb{R}) .
\end{aligned}
$$

Here we assume that $B:[0, T] \times H \rightarrow H$ and $C:[0, T] \times H \rightarrow L(H, H)$ are continuous (we assume continuity of $B$ and $\frac{\partial F}{\partial t}$ for simplicity of exposition, but this detail can be generalized). Since

$$
G(t, x):=\frac{\partial F}{\partial t}(t, x)+\langle A x+B(t, x), D F(t, x)\rangle, \quad x \in D(A), t \in[0, T]
$$

satisfies

$$
G(t, x)=-\frac{1}{2} \operatorname{Tr}\left(C(t, x) Q C^{*}(t, x) D^{2} F(t, X(t))\right)
$$


then it has a continuous extension on $[0, T] \times H$ and Theorem 1 is applicable, if $(X(t))_{t \in\left[t_{0}, T\right]}$ (for some $t_{0} \in[0, T)$ ) is a continuous process in $H$ satisfying

$$
X(t)=e^{\left(t-t_{0}\right) A} x^{0}+\int_{t_{0}}^{t} e^{(t-s) A} B(s, X(s)) \mathrm{d} s+\int_{t_{0}}^{t} e^{(t-s) A} C(s, X(s)) \mathrm{d} W(s),
$$

thus we get

$$
F(t, X(t))=F\left(t_{0}, x^{0}\right)+\int_{t_{0}}^{t}\langle D F(s, X(s)), C(s, X(s)) \mathrm{d} W(s)\rangle .
$$

We can now easily prove the following uniqueness result. We do not repeat the assumptions on $H, W, e^{t A}, B$.

Theorem 3 Assume that for every $\left(t_{0}, x^{0}\right) \in[0, T] \times H$, there exists at least one continuous process $X$ in $H$ satisfying Eq. (20). Then the following holds.

(i) The Kolmogorov Eq. (18) has a unique solution in the class of bounded functions $F$ satisfying (19).

(ii) If $C \in C_{b}([0, T] \times H ; L(H, H))$, it has a unique solution in the class of functions $F$ satisfying (19) and $\|D F\|_{\infty}<\infty$.

Proof (i) The stochastic integral $\int_{t_{0}}^{t}\langle D F(s, X(s)), C(s, X(s)) \mathrm{d} W(s)\rangle$ is a local martingale. Since $F$ is bounded, from identity (21) it follows that it is uniformly integrable, and hence, it is also a martingale. Therefore, taking expectation in (21), we get $F\left(t_{0}, x^{0}\right)=E[\varphi(X(T))]$, formula which identifies $F$, since $t_{0}$ and $x^{0}$ are arbitrary.

(ii) If $C$ and $D F$ are bounded, the stochastic integral in (21) is a martingale. We conclude as in i).

\section{Application to Path-Dependent Functionals}

We will now apply the abstract results of Sect. 3 to obtain an Itô formula for pathdependent functionals of continuous processes, stated in Theorem 4 . In the first sections we will introduce the necessary spaces and operators and we will show that the infinite-dimensional reformulation of path-dependent problems appears naturally when dealing with path-dependent SDEs (see again [13] for a more detailed discussion).

\subsection{Infinite-Dimensional Formulation of Itô Processes}

In this and the following sections we will denote by $C_{t}$ the space of $\mathbb{R}^{d}$-valued functions on $[0, t]$ that can have a jump only at $t$, that is

$$
C_{t}=\left\{\varphi:[0, t] \rightarrow \mathbb{R}^{d}: \varphi \in C\left([0, t) ; \mathbb{R}^{d}\right), \exists \lim _{s \rightarrow t^{-}} \varphi(s) \in \mathbb{R}^{d}\right\}
$$


endowed with the supremum norm. The space $C_{t}$ is clearly isomorphic to the product space

$$
\mathbb{R}^{d} \times\left\{\varphi \in C\left([0, t) ; \mathbb{R}^{d}\right), \exists \lim _{s \rightarrow t^{-}} \varphi(s) \in \mathbb{R}^{d}\right\} .
$$

Let $y(t)$ be the continuous process in $\mathbb{R}^{d}$ given by

$$
y(t)=y^{0}+\int_{0}^{t} b(s) \mathrm{d} s+\int_{0}^{t} c(s) \mathrm{d} W(s)
$$

where $b$ and $c$ are progressively measurable processes, with values in $\mathbb{R}^{d}$ and $\mathbb{R}^{k \times d}$, respectively, such that

$$
\int_{0}^{T}|b(s)| \mathrm{d} s<\infty, \quad \int_{0}^{T}\|c(s)\|^{2} \mathrm{~d} s<\infty
$$

and $y^{0}$ is a $\mathscr{F}_{0}$-measurable random vector.

Let us introduce the following infinite-dimensional reformulation. We will work in the space

$$
E=\mathbb{R}^{d} \times\left\{\varphi \in C\left([-T, 0) ; \mathbb{R}^{d}\right): \exists \lim _{s \rightarrow 0^{-}} \varphi(s) \in \mathbb{R}^{d}\right\},
$$

whose elements we shall usually denote by $x=\left(\begin{array}{l}x_{1} \\ x_{2}\end{array}\right) . E$ is a Banach space when endowed with the norm $\left\|\left(\begin{array}{l}x_{1} \\ x_{2}\end{array}\right)\right\|^{2}=\left|x_{1}\right|^{2}+\left\|x_{2}\right\|_{\infty}^{2}$; the notation $\langle\cdot, \cdot\rangle$ will denote the duality pairing between $E$ and its dual space $E^{*}$. The space $E$ is densely and continuously embedded in the product space

$$
H=\mathbb{R}^{d} \times L^{2}\left(-T, 0 ; \mathbb{R}^{d}\right)
$$

We also introduce the unbounded linear operator $A: D(A) \subset H \rightarrow H$ defined as

$$
\begin{aligned}
D(A) & =\left\{\left(\begin{array}{l}
x_{1} \\
x_{2}
\end{array}\right) \in H: x_{2} \in W^{1,2}\left(-T, 0 ; \mathbb{R}^{d}\right), x_{1}=\lim _{s \rightarrow 0^{-}} x_{2}(s)\right\}, \\
A & =\left(\begin{array}{ll}
0 & 0 \\
0 & \frac{d}{d r}
\end{array}\right),
\end{aligned}
$$

where we identify an element in $W^{1,2}$ with the restriction of its continuous version to $[-T, 0)$. Therefore, we identify also the space

$$
\widetilde{E}=\overline{D(A)}^{E}=\left\{y=\left(\begin{array}{l}
x_{1} \\
x_{2}
\end{array}\right) \in E: x_{1}=\lim _{s \rightarrow 0^{-}} x_{2}(s)\right\} .
$$

The operator $A$ generates a strongly continuous semigroup $e^{t A}$ in $H$. This semigroup turns out to be not strongly continuous in $E$; nevertheless $e^{t A}$ maps $\widetilde{E}$ in itself and is 
strongly continuous in $\widetilde{E}$. This follows from the fact that the semigroup $e^{t A}$ has the explicit form

$$
e^{t A} x=\left(\begin{array}{c}
x_{1} \\
x_{2}(\cdot+t) \mathbb{1}_{[-T,-t)}+x_{1} \mathbb{1}_{[-t, 0)}
\end{array}\right)
$$

(see [1] for details on the operator $A$ in the context of delay equations and [13] about its role in the theory of path-dependent equations).

For any $t \in[0, T]$ we introduce the operator

$$
L^{t}: C_{t} \rightarrow E
$$

defined as

$$
L^{t} \gamma=\left(\begin{array}{c}
\gamma(t) \\
\gamma(0) \mathbb{1}_{[-T,-t)}+\gamma(t+\cdot) \mathbb{1}_{[-t, 0)}
\end{array}\right)
$$

for every $\gamma \in C_{t}$.

Using (28), it is easy to show (see also Proposition 3) that

$$
X(t)=L^{t} y_{t},
$$

as a $H$-valued process, is given by

$$
X(t)=e^{t A} X^{0}+\int_{0}^{t} e^{(t-s) A} B(s) \mathrm{d} s+\int_{0}^{t} e^{(t-s) A} C(s) \mathrm{d} W(s),
$$

where $X^{0}=\left(\begin{array}{c}x^{0} \\ x^{0} \mathbb{1}_{[-T, 0)}\end{array}\right)$ and the processes $B:[0, T] \rightarrow E$ and $C:[0, T] \rightarrow$ $L\left(\mathbb{R}^{k}, E\right)$ are given by

$$
B(t)=\left(\begin{array}{c}
b(s) \\
0
\end{array}\right), \quad C(s) u=\left(\begin{array}{c}
c(s) u \\
0
\end{array}\right) \text { for } u \in \mathbb{R}^{k} .
$$

The validity of (30) corresponds to saying that $X$ is the unique mild solution to the linear equation

$$
\mathrm{d} X(t)=A X(t) \mathrm{d} t+B(t) \mathrm{d} t+C(t) \mathrm{d} W(t) ;
$$

hence, we see that our infinite-dimensional reformulation forces us to deal with equations even if we start from finite-dimensional processes: the operator $A$ appears as a consequence of the introduction of the second component that represents the "past trajectory" of the process (see Remark 3).

Proposition 2 The process $X$ is such that $X(t) \in \widetilde{E}$ for every $t$ and the trajectories $t \mapsto X(t)$ are almost surely continuous as maps from $[0, T]$ to $\widetilde{E}$.

Proof The random variable $X^{0}$ takes values in $\widetilde{E}$ by definition. Since the process $y$ has almost surely continuous trajectories, $\left(L^{t} y_{t}\right)_{2} \in C\left([-T, 0) ; \mathbb{R}^{d}\right)$ and $L^{t} y_{t}$ belongs to $\widetilde{E}$. To check the almost sure continuity of the trajectories of $X$ as a $\widetilde{E}$-valued process denote again by $\Omega_{0} \subset \Omega$ a null set such that $t \mapsto y(\omega, t)$ is continuous for every $\omega \in \Omega \backslash \Omega_{0}$, fix $\omega \in \Omega \backslash \Omega_{0}$, fix $t, s \in[0, T]$ and $\varepsilon>0$; we can suppose $t>s$ 
without loss of generality. Since $y(\omega, \cdot)$ is uniformly continuous on $[0, T]$ we can find $\delta$ such that $|y(t)-y(s)|<\varepsilon / 2$ if $t-s<\delta$. Then for $t-s<\delta$

$$
\begin{aligned}
\|X(t)-X(s)\|_{\widetilde{E}} \leq & |y(t)-y(s)| \\
& +\max \left\{\sup _{r \in[0, t-s]}|y(0)-y(r)|, \sup _{r \in[0, s]}|y(t-s+r)-y(r)|\right\} \\
\leq & \varepsilon .
\end{aligned}
$$

\subsection{Infinite-Dimensional Formulation of Path-Dependent Functionals}

A path-dependent functional $f$ is a family of functionals $f(t, \cdot), t \in[0, T]$, such that

$$
f(t, \cdot): C_{t} \rightarrow \mathbb{R}
$$

Among the examples of path-dependent functional, let us mention the integral ones

$$
f(t, \gamma)=\int_{0}^{t} g(\gamma(t), \gamma(s)) \mathrm{d} s
$$

and those involving pointwise evaluations, like for instance

$$
f(t, \gamma)=q\left(\gamma(t), \gamma\left(t_{0}\right)\right) \mathbb{1}_{t>t_{0}}
$$

Here we assume that $g: \mathbb{R}^{d} \times \mathbb{R}^{d} \rightarrow \mathbb{R}$ is a measurable function in the first example, with $|g(a)| \leq C\left(1+|a|^{2}\right)$ and that $q: \mathbb{R}^{d} \times \mathbb{R}^{d} \rightarrow \mathbb{R}$ is a measurable function in the second example and $t_{0} \in[0, T]$ is a given point. In order to apply Itô calculus let us simplify and assume that $g$ and $q$ are twice continuously differentiable with bounded derivatives of all orders.

Given a path-dependent functional $f(t, \cdot), t \in[0, T]$ we may associate to it a map $F:[0, T] \times E \rightarrow \mathbb{R}$ setting

$$
F(t, x)=f\left(t, M_{t} x\right)
$$

where

$$
M_{t}: E \rightarrow C_{t}
$$

is defined as

$$
M_{t} x(s)=x_{2}(s-t) \mathbb{1}_{[0, t)}(s)+x_{1} \mathbb{1}_{\{t\}}(s), \quad s \in[0, t] .
$$

Remark 2 Notice that $M_{t} L^{t}$ is the identity on $C_{t}$. 
Here we see that if $f$ were defined only on $C\left([0, t] ; \mathbb{R}^{d}\right)$, then $F$ would be defined on $[0, T] \times \widetilde{E}$, because of the definition of the operators $M_{t}$; our abstract results require instead $F$ to be defined on $[0, T] \times E$; see Remark 1 .

Path-dependent functionals are often studied in spaces of càdlàg paths. The framework presented here can be easily modified to do so, similarly to what is done in [13]; however, this would require the introduction of further spaces, thus complicating notations, but would not lead to generalizations of the result proved here.

The aim of this section is to show that Examples (33) and (34) fulfill the assumptions of Theorem 2.

The abstract reformulation of the functional given in (33) is the map $F:[0, T] \times$ $E \rightarrow \mathbb{R}$ defined as

$$
\begin{aligned}
F(t, x)=\int_{0}^{t} g\left(M_{t} x(t), M_{t} x(s)\right) \mathrm{d} s & =\int_{0}^{t} g\left(x_{1}, x_{2}(s-t)\right) \mathrm{d} s \\
& =\int_{-t}^{0} g\left(x_{1}, x_{2}(r)\right) d r .
\end{aligned}
$$

Hence,

$$
\begin{aligned}
\frac{\partial F}{\partial t}(t, x) & =g\left(x_{1}, x_{1}\right)-\int_{0}^{t} D g\left(x_{1}, x_{2}(s-t)\right) \cdot x_{2}^{\prime}(s-t) \mathrm{d} s \\
& =g\left(x_{1}, x_{1}\right)-\int_{-t}^{0} g\left(x_{1}, x_{2}(r)\right) \cdot x_{2}^{\prime}(r) \mathrm{d} r,
\end{aligned}
$$

which is meaningful, for example, if $x_{2}$ belongs to $C^{1}\left([-T, 0] ; \mathbb{R}^{d}\right)$. Indeed since $A(D(A)) \subset\{0\} \times L^{2}\left(-T, 0 ; \mathbb{R}^{d}\right)$, we have

$$
\widetilde{D}=A^{-1}(E)=A^{-1}\left(\{0\} \times E_{2}\right),
$$

where

$$
E_{2}=\left\{\varphi \in C\left([-T, 0) ; \mathbb{R}^{d}\right): \exists \lim _{s \rightarrow 0^{-}} \varphi(s) \in \mathbb{R}^{d}\right\}
$$

and so

$$
\widetilde{D}=\left\{\left(\begin{array}{l}
x_{1} \\
x_{2}
\end{array}\right) \in D(A): x_{2} \in C^{1}\left([-T, 0) ; \mathbb{R}^{d}\right)\right\} .
$$

Moreover, the time derivative of $F$ is defined for every $t \in[0, T]$. Therefore, we see that

$$
\frac{\partial F}{\partial t}:[0, T] \times \widetilde{D} \rightarrow \mathbb{R}
$$

is a natural assumption, while $\frac{\partial F}{\partial t}:[0, T] \times E \rightarrow \mathbb{R}$ would not be. Since $g$ is continuous we also have that $\partial_{t} F$ belongs to $C([0, T] \times \widetilde{D} ; \mathbb{R})$. 
Let us then investigate the function

$$
G(t, x):=\frac{\partial F}{\partial t}(t, x)+\langle A x, D F(t, x)\rangle \quad x \in \widetilde{D}, t \in[0, T] .
$$

For $h \in E$ we have

$$
\begin{aligned}
\langle h, D F(s, x)\rangle & =\lim _{\varepsilon \rightarrow 0} \frac{1}{\varepsilon} \int_{0}^{t}\left(g\left(x_{1},\left(x_{2}+\varepsilon h_{2}\right)(s-t)\right)-g\left(x_{1}, x_{2}(s-t)\right)\right) \mathrm{d} s \\
& =\int_{0}^{t} D g\left(x_{1}, x_{2}(s-t)\right) \cdot h_{2}(s-t) \mathrm{d} s \\
& =\int_{-t}^{0} D g\left(x_{1}, x_{2}(r)\right) \cdot h_{2}(r) d r .
\end{aligned}
$$

But then

$$
G(s, x)=g\left(x_{1}, x_{1}\right) ;
$$

thus we see that the function $G(s, x)$ is well defined on $E$ too! The assumption $G \in C([0, T] \times \widetilde{E})$ is fulfilled.

The abstract reformulation of the functional given in (34) is the map $F: E \rightarrow \mathbb{R}$ defined as

$$
F(t, x)=q\left(M_{t} x(t), M_{t} x\left(t_{0}\right)\right) \mathbb{1}_{t>t_{0}}=q\left(x_{1}, x_{2}\left(t_{0}-t\right)\right) \mathbb{1}_{t>t_{0}} .
$$

Hence, writing $\partial_{1} q$ and $\partial_{2} q$ for the derivatives of $q$ with respect to its first and second variable, respectively, for $t \neq t_{0}$,

$$
\frac{\partial F}{\partial t}(t, x)=-\partial_{2} q\left(x_{1}, x_{2}\left(t_{0}-t\right)\right) \cdot x_{2}^{\prime}\left(t_{0}-t\right) \mathbb{1}_{t>t_{0}}
$$

which requires $x_{2} \in C^{1}$.

But

$$
\begin{aligned}
G(t, x)= & -\partial_{2} q\left(x_{1}, x_{2}\left(t_{0}-t\right)\right) \cdot x_{2}^{\prime}\left(t_{0}-t\right) \mathbb{1}_{t>t_{0}} \\
& +\partial_{1} q\left(x_{1}, x_{2}\left(t_{0}-t\right)\right) \mathbb{1}_{t>t_{0}} \cdot(A x)_{1}+\partial_{2} q\left(x_{1}, x_{2}\left(t_{0}-t\right)\right) \mathbb{1}_{t>t_{0}} \\
& \cdot(A x)_{2}\left(t_{0}-t\right) \\
= & 0
\end{aligned}
$$

because $(A x)_{1}=0$ and $(A x)_{2}\left(t_{0}-t\right)=x_{2}^{\prime}\left(t_{0}-t\right)$. Again, $G$ extends continuously to $E$.

\subsection{Infinite-Dimensional Formulation of Path-Dependent SDEs}

In Sect. 5.1 we have formulated a classical Itô process as an infinite-dimensional process given by a mild formula; this apparently not natural formulation is suggested 
by the case when the process is the solution of a path-dependent SDE. For these equations, the mild formulation is natural, due to the similarity with delay equations, where the infinite-dimensional approach is classical. Let us give here some details about the case of a path-dependent SDE.

Let $(\Omega, \mathscr{F}, \mathbb{P})$ be a complete probability space, $\mathbb{F}=\left(\mathscr{F}_{t}\right)_{t \geq 0}$ a complete filtration, $(W(t))_{t \geq 0}$ a Brownian motion in $\mathbb{R}^{k}$ (we shall write $W^{i}(t)$ for its coordinates), $y^{0}$ an $\mathscr{F}_{0}$-measurable random vector of $\mathbb{R}^{d}$. Consider the path-dependent SDE in $\mathbb{R}^{d}$

$$
d y(t)=b\left(t, y_{t}\right) \mathrm{d} t+\sigma\left(t, y_{t}\right) \mathrm{d} W(t), \quad y(0)=y^{0} .
$$

The solution $(y(t))_{t \in[0, T]}$ is a stochastic process in $\mathbb{R}^{d}$ and $y_{t}$ denotes the window

$$
y_{t}:=\{y(s)\}_{s \in[0, t]} .
$$

About $b$ and $\sigma$, initially we assume that, for each $t \in[0, T]$, the function $b(t, \cdot)$ maps $C\left([0, t] ; \mathbb{R}^{d}\right)$ into $\mathbb{R}^{d}$ and the function $\sigma(t, \cdot)$ maps $C\left([0, t] ; \mathbb{R}^{d}\right)$ into $\mathbb{R}^{k \times d}$; moreover, we assume that $b$ ad $\sigma$ are locally bounded measurable functions, for each $t \in[0, T]$, with bounds uniform in $t$ and that the processes $b\left(t, y_{t}\right)$ and $\sigma\left(t, y_{t}\right)$ are progressively measurable. These are relatively weak requirements to give a meaning to the integral equation

$$
y(t)=y^{0}+\int_{0}^{t} b\left(s, y_{s}\right) \mathrm{d} s+\int_{0}^{t} \sigma\left(s, y_{s}\right) \mathrm{d} W(s) .
$$

If, in addition, we assume that $b(t, \cdot)$ and $\sigma(t, \cdot)$ are Lipschitz continuous from $C\left([0, t] ; \mathbb{R}^{d}\right)$ to $\mathbb{R}^{d}$ and $\mathbb{R}^{k \times d}$, respectively, with Lipschitz constants independent of $t$, then existence and uniqueness of a continuous solution, adapted to the completed filtration of $W$, holds true. We shall also write $\sigma\left(t, y_{t}\right) \mathrm{d} W(t)$ as $\sum_{i=1}^{k} \sigma_{i}\left(t, y_{t}\right) \mathrm{d} W^{i}(t)$.

We take the operator $A$ as in Sect. 5.1 and we define the continuous nonlinear operators $B:[0, T] \times \widetilde{E} \rightarrow E, C_{i}:[0, T] \times \widetilde{E} \rightarrow E, i=1, \ldots, k$, as

$$
\begin{aligned}
B(t, x) & =\left(\begin{array}{c}
b\left(t, M_{t} x\right) \\
0
\end{array}\right) \\
C_{i}(t, x) & =\left(\begin{array}{c}
\sigma_{i}\left(t, M_{t} x\right) \\
0
\end{array}\right) .
\end{aligned}
$$

Finally, we set $U=\mathbb{R}^{k}$, take $Q$ equal to the identity in $U$ and consider, for every $(t, x) \in[0, T] \times E$, the bounded linear operator $C(t, x): U \rightarrow E$ having components $C_{i}(t, x)$.

Given a $\mathscr{F}_{0}$-measurable random variable $X^{0}$ with values in $E$ we may now formulate the path-dependent SDE in the Banach space $E$, i.e.,

$$
\mathrm{d} X(t)=(A X(t)+B(t, X(t))) \mathrm{d} t+C(t, X(t)) \mathrm{d} W(t), \quad X(0)=X^{0} .
$$

The natural concept of solution here would be that of mild solution, but since under our assumptions the stochastic convolution is a priori well defined only in $H$, we consider 
Eq. (40) in its mild form in the space $H$, that is

$$
X(t)=e^{t A} X^{0}+\int_{0}^{t} e^{(t-s) A} B(s, X(s)) \mathrm{d} s+\int_{0}^{t} e^{(t-s) A} C(s, X(s)) \mathrm{d} W(s) .
$$

Notice that since $E$ is continuously embedded in $H$ all the integrals in (41) are meaningful.

In the following proposition we clarify the relation between Eqs. (41) and (37).

Proposition 3 Given an $\mathscr{F}_{0}$-measurable random vector $y^{0}$ of $\mathbb{R}^{d}$, set $X^{0}=$ $\left(y^{0}, y^{0} \mathbb{1}_{[-T, 0)}\right)^{T}$. Then, if $\{y(t)\}_{t \in[0, T]}$ is a solution to Eq. (37), the process

$$
X(t)=L^{t} y_{t}
$$

is a solution to Eq. (41). We also have

$$
y_{t}=M_{t} X(t)
$$

Proof By (28) the first component of Eq. (41) reads

$$
\begin{aligned}
\left(L^{t} y_{t}\right)_{1}=y(t) & =X_{1}(t)=X_{1}^{0}+\int_{0}^{t} B(s, X(s))_{1} \mathrm{~d} s+\int_{0}^{t} C(s, X(s))_{1} \mathrm{~d} W(s) \\
& =y^{0}+\int_{0}^{t} b\left(s, M_{s} X(s)\right) \mathrm{d} s+\int_{0}^{t} \sigma\left(s, M_{s} X(s)\right) \mathrm{d} W(s) \\
& =y^{0}+\int_{0}^{t} b\left(s, M_{s} L^{s} y_{s}\right) \mathrm{d} s+\int_{0}^{t} \sigma\left(s, M_{s} L^{s} y_{s}\right) \mathrm{d} W(s) \\
& =y^{0}+\int_{0}^{t} b\left(s, y_{s}\right) \mathrm{d} s+\int_{0}^{t} \sigma\left(s, y_{s}\right) \mathrm{d} W(s)
\end{aligned}
$$

which holds true because it is Eq. (37). About the second component, we have

$$
\begin{aligned}
\left(L^{t} y_{t}\right)_{2}(r)= & X_{2}(t)(r)=X_{2}^{0}(r+t) 1_{[-T,-t]}(r)+X_{1}^{0} 1_{[-t, 0]}(r) \\
& +\int_{0}^{t} b\left(s, M_{s} X(s)\right) 1_{[-t+s, 0]}(r) \mathrm{d} s \\
& +\int_{0}^{t} \sigma\left(s, M_{s} X(s)\right) 1_{[-t+s, 0]}(r) \mathrm{d} W(s) \\
= & y^{0}+\int_{0}^{t} b\left(s, y_{s}\right) 1_{[-t+s, 0]}(r) \mathrm{d} s+\int_{0}^{t} \sigma\left(s, y_{s}\right) 1_{[-t+s, 0]}(r) \mathrm{d} W(s) .
\end{aligned}
$$

For $r \in[-T,-t]$ this identity reads $y^{0}=y^{0}$, which is true. For $r \in[-t, 0]$ we have

$$
y(t+r)=y_{t}(t+r)=y^{0}+\int_{0}^{t+r} b\left(s, y_{s}\right) \mathrm{d} s+\int_{0}^{t+r} \sigma\left(s, y_{s}\right) \mathrm{d} W(s),
$$


because $\mathbb{1}_{[-t+s, 0]}(r)=0$ for $s \in[t+r, t]$. This is again a copy of Eq. (37). The proof is complete.

Remark 3 We have seen that, at the level of the mild formulation, the equation in Hilbert space is just given by two copies of the original SDE. On the contrary, at the level of the differential formulation, we formally have

$$
\begin{aligned}
\mathrm{d} X_{1}(t) & =B\left(t, M_{t} X(t)\right) \mathrm{d} t+C_{i}\left(t, M_{t} X(t)\right) \mathrm{d} W(t) \\
\mathrm{d} X_{2}(t) & =\frac{d}{d r} X_{2}(t) \mathrm{d} t .
\end{aligned}
$$

The first equation, again, is a rewriting of the path-dependent SDE. But the second equation is just a consistency equation, necessary since we need to introduce the component $X_{2}(t)$. Here we see one of the technical problems which motivate this paper: $X_{2}(t)=\left(L^{t} y_{t}\right)_{2}$ is "never" differentiable (being a trajectory of solution of the $\mathrm{SDE}$, it has the level of regularity of Brownian motion). In other words, $X_{2}(t)$ "never" belongs to $D(A)$.

\subsection{Itô Formula for Path-Dependent Functionals}

Having introduced the previous infinite-dimensional reformulations, we can apply our abstract result of Sect. 3 to obtain a Itô formula for path-dependent functionals of continuous paths. To this end we recall that we intend to apply Theorem 2 to the spaces

$$
\begin{aligned}
H_{1} & =\mathbb{R}^{d} \\
E_{2} & =\left\{\varphi \in C\left([-T, 0) ; \mathbb{R}^{d}\right): \exists \lim _{s \rightarrow 0^{-}} \varphi(s) \in \mathbb{R}^{d}\right\}, \\
H_{2} & =L^{2}\left(-T, 0 ; \mathbb{R}^{d}\right), \\
E & =H_{1} \times E_{2}, \\
H & =H_{1} \times H_{2}, \\
\widetilde{E} & =\left\{\left(\begin{array}{l}
x_{1} \\
x_{2}
\end{array}\right) \in E: x_{1}=\lim _{s \rightarrow 0^{-}} x_{2}(s)\right\}, \\
\widetilde{D} & =\left\{\left(\begin{array}{l}
x_{1} \\
x_{2}
\end{array}\right) \in E: x_{2} \in C^{1}\left([-T, 0) ; \mathbb{R}^{d}\right), x_{1}=\lim _{s \rightarrow 0^{-}} x_{2}(s)\right\}, \\
U & =\mathbb{R}^{k}
\end{aligned}
$$

and to the operator $A$ on $H$ given by

$$
A\left(\begin{array}{l}
x_{1} \\
x_{2}
\end{array}\right)=\left(\begin{array}{c}
0 \\
\dot{x}_{2}
\end{array}\right)
$$

on the domain

$$
D(A)=\left\{\left(\begin{array}{l}
x_{1} \\
x_{2}
\end{array}\right) \in \mathbb{R}^{d} \times W^{1,2}\left(-T, 0 ; \mathbb{R}^{d}\right): x_{2}(0)=x_{1}\right\}
$$


As before $y$ is a continuous process in $\mathbb{R}^{d}$ given by

$$
y(t)=y^{0}+\int_{0}^{t} b(s) \mathrm{d} s+\int_{0}^{t} c(s) \mathrm{d} W(s),
$$

where $W, b$ and $c$ are as in Sect. 5.1 (we set $Q=I d_{\mathbb{R}^{k}}$ ) and we set

$$
X(t)=L^{t} y_{t}
$$

Theorem 4 Let $\{f(t, \cdot)\}_{t \in[0, T]}, f(t, \cdot): C_{t} \rightarrow \mathbb{R}$, be a path-dependent functional and define

$$
\begin{aligned}
& F:[0, T] \times E \longrightarrow \mathbb{R} \\
& F(t, x)=f\left(t, M_{t} x\right) .
\end{aligned}
$$

\section{Suppose that}

(i) $F \in C([0, T] \times E ; \mathbb{R})$;

(ii) $F$ is twice differentiable in its second variable with $D F \in C\left([0, T] \times E ; E^{*}\right)$ and $D^{2} F \in C\left([0, T] \times E ; L\left(E ; E^{*}\right)\right)$;

(iii) there exists a set $\mathscr{T} \subset[0, T]$ such that $\lambda(\mathscr{T})=T$ and $F$ is differentiable with respect to $t$ on $\mathscr{T} \times \widetilde{D}$;

(iv) there exists a continuous function $G:[0, T] \times \widetilde{E} \rightarrow \mathbb{R}$ such that

$$
G(t, x)=\frac{\partial F}{\partial t}(t, x)+\langle A x, D F(t, x)\rangle
$$

for $(t, x) \in \mathscr{T} \times \widetilde{D}$.

Then the identity

$$
\begin{aligned}
f\left(t, y_{t}\right)= & f\left(0, y_{0}\right)+\int_{0}^{t} G(s, X(s)) \mathrm{d} s \\
& +\int_{0}^{t}\left(\langle B(s), D F(s, X(s))\rangle+\frac{1}{2} \operatorname{Tr}_{\mathbb{R}^{d}}\left[C(s) C(s)^{*} D^{2} F(s, X(s))\right]\right) \mathrm{d} s \\
& +\int_{0}^{t}\langle D F(s, X(s)), C(s) \mathrm{d} W(s)\rangle
\end{aligned}
$$

holds in probability.

Proof First notice that by Proposition 2 and the discussion in Sect. 5.1 the process $X$ has continuous paths in $\widetilde{E}$, therefore the set $\{X(t)\}_{t \in[0, T]}$ is a compact set in $E$. With this choice of $E$ and $H$, a sequence $\mathscr{J}_{n}: H \rightarrow E$ satisfying the requirements of Theorem 2 can be constructed (following [13]) in this way: for any $\varepsilon \in(0, T / 2)$ define the function $\tau_{\varepsilon}:[-T, 0] \rightarrow[-T, 0]$ as 


$$
\tau_{\varepsilon}(x)= \begin{cases}-T+\varepsilon & \text { if } x \in[-T,-T+\varepsilon] \\ x & \text { if } x \in[-T+\varepsilon,-\varepsilon] \\ -\varepsilon & \text { if } x \in[-\varepsilon, 0] .\end{cases}
$$

Then choose any $C^{\infty}(\mathbb{R} ; \mathbb{R})$ function $\rho$ such that $\|\rho\|_{1}=1,0 \leq \rho \leq 1$ and $\operatorname{supp}(\rho) \subseteq$ $[-1,1]$ and define a sequence $\left\{\rho_{n}\right\}$ of mollifiers by $\rho_{n}(x):=n \rho(n x)$. Set, for any $\varphi \in L^{2}\left(-T, 0 ; \mathbb{R}^{d}\right)$

$$
\mathscr{J}_{n} \varphi(x):=\int_{-T}^{0} \rho_{n}\left(\rho_{2 n} * \tau_{\frac{1}{n}}(x)-y\right) \varphi(y) \mathrm{d} y
$$

and finally define $\mathscr{J}_{n}$ as

$$
\mathscr{J}_{n}\left(\begin{array}{l}
x \\
\varphi
\end{array}\right):=\left(\begin{array}{c}
x \\
\mathscr{J}_{n} \varphi
\end{array}\right) .
$$

The proof is then completed applying Theorem 2 to the function $F$ and its extension $G$.

Remark 4 The choice of the spaces $H$ and $E$ and of the operators $A$ and $\mathscr{J}_{n}$ does not depend on $F$ and is the same for all path-dependent functionals of continuous processes. The only assumptions that need to be checked on each functional are the regularity conditions and existence of the extension $G$.

The path-dependent functional given in (34) is not covered by the previous result since it is not jointly continuous on $[0, T] \times E$. However, it satisfies the assumptions of Corollary 1 , which we now state in its path-dependent formulation.

Corollary 2 Let $f$ and $F$ be as in Theorem 4. If $F$ satisfies the assumptions of Corollary 1 , then the formula

$$
\begin{aligned}
f\left(T, y_{T}\right)= & F\left(0, y_{0}\right)+\sum_{j=1}^{n}\left[f\left(t_{j}, y_{t_{j}}\right)-f\left(t_{j}-, y_{t_{j}}\right)\right] \\
& +\int_{0}^{T} G(s, X(s)) \mathrm{d} s+\int_{0}^{T}\langle D F(s, X(s)), C(s) \mathrm{d} W(s)\rangle \\
& +\int_{0}^{T}\left(\langle B(s), D F(s, X(s))\rangle+\frac{1}{2} \operatorname{Tr}_{H_{1}}\left[C(s) Q C(s)^{*} D^{2} F(s, X(s))\right]\right) \mathrm{d} s
\end{aligned}
$$

holds in probability.

\section{Application to Kolmogorov Equations}

\subsection{Uniqueness of Solutions}

We begin investigating Kolmogorov equation in the abstract setting of Sect. 3, discussing the particular case of path-dependent Kolmogorov equations afterward. Let 
the spaces $H, E, \widetilde{E}, \widetilde{D}$ and $U$, the Wiener process $W$ and the operator $A$ be as in Sect. 3; given $B:[0, T] \times \widetilde{E} \rightarrow E$ and $C:[0, T] \times \widetilde{E} \rightarrow L\left(U ; H_{1} \times\{0\}\right)$ we can consider the partial differential equation

$$
\left\{\begin{array}{l}
\frac{\partial V}{\partial t}(t, x)+\langle D V(t, x), A x+B(t, x)\rangle+\frac{1}{2} \operatorname{Tr}_{H_{1}}\left(C(s, x) Q C(s, x)^{*} D^{2} V(t, x)\right)=0 \\
V(T, \cdot)=\Phi
\end{array}\right.
$$

where the terminal condition $\Phi$ is chosen in $C_{b}^{2, \alpha}(E)$, the space of twice differentiable real-valued functions $\varphi$ on $E$ such that $\varphi, D \varphi$ and $D^{2} \varphi$ are bounded and the map $E \ni x \rightarrow D^{2} \varphi(x) \in L\left(E ; E^{*}\right)$ is $\alpha$-Hölder continuous.

Definition 1 We say that a function $V:[0, T] \times E \rightarrow \mathbb{R}$ is a classical solution to Eq. (45) in $E$ if

$$
V \in L^{\infty}\left(0, T ; C_{b}^{2, \alpha}(E ; \mathbb{R})\right) \cap C([0, T] \times E ; \mathbb{R})
$$

$V$ is differentiable with respect to $t$ on $\mathscr{T} \times \widetilde{D}, \mathscr{T} \subset[\underset{D}{[0}, T]$ being a set of full measure, and satisfies identity (45) for every $t \in \mathscr{T}$ and $x \in \widetilde{D}$.

Assume that $B$ and $C$ are continuous and such that the stochastic SDE

$$
\mathrm{d} X(s)=A X(s)+B(s, X(s)) \mathrm{d} s+C(s, X(s)) \mathrm{d} W(s) \text { for } s \in[t, T], \quad X(t)=x
$$

has a mild solution $X^{t, x}$ in $H$ for all $t \in[0, T]$ and all $x \in \widetilde{E}$, such that $X^{t, x}(s)$ belongs to $\widetilde{E}$ for all $s \in[t, T]$ and that the set $\left\{X^{t, x}(s)\right\}_{s \in[t, T]}$ is almost surely relatively compact in $E$.

Theorem 5 Under the above assumptions any classical solution to Eq. (45) is uniquely determined on the space $\widetilde{E}$

Proof Suppose there exists a solution $V$. Since $D V, D^{2} V, B$ and $C$ are defined on $[0, T] \times \widetilde{E}$ and are continuous, the function

$$
G(t, x)=-\langle B(t, x), D V(t, x)\rangle-\frac{1}{2} \operatorname{Tr}_{\mathbb{R}^{d}}\left[C(t, x) C(t, x)^{*} D^{2} V(t, x)\right]
$$

is a continuous extension of

$$
\frac{\partial V}{\partial t}(t, x)+\langle A x, D V(t, x)\rangle
$$

from $\mathscr{T} \times \widetilde{D}$ to $[0, T] \times \widetilde{E}$, because $V$ satisfies Kolmogorov equation. 
Therefore, we can apply Theorem 2 to obtain

$$
\begin{aligned}
\Phi\left(X^{t, x}(T)\right)= & V\left(t, X^{t, x}(t)\right)+\int_{t}^{T} G\left(s, X^{t, x}(s)\right) \mathrm{d} s \\
& +\int_{t}^{T}\left\langle B\left(s, X^{t, x}(s)\right), D V\left(s, X^{t, x}(s)\right)\right\rangle \mathrm{d} s \\
& +\frac{1}{2} \int_{t}^{T} \operatorname{Tr}_{H_{1}}\left[C\left(s, X^{t, x}(s)\right) C\left(s, X^{t, x}(s)\right)^{*} D^{2} V\left(s, X^{t, x}(s)\right)\right] \mathrm{d} s \\
& +\int_{t}^{T}\left\langle D V\left(s, X^{t, x}(s)\right), C\left(s, X^{t, x}(s)\right) \mathrm{d} W(s)\right\rangle \\
= & V\left(t, X^{t, x}(t)\right)+\int_{t}^{T}\left\langle D V\left(s, X^{t, x}(s)\right), C\left(s, X^{t, x}(s)\right) \mathrm{d} W(s)\right\rangle .
\end{aligned}
$$

The integral in the last line is actually a stochastic integral in a Hilbert space, since for every $u \in U C(s, x) u$ belongs to $H_{1} \times\{0\}$; taking expectations in the previous identity we obtain that

$$
V(t, x)=\mathbb{E}\left[\Phi\left(X^{t, x}(T)\right)\right] .
$$

In the path-dependent case the above discussion can be rephrased as follows. Choose the spaces $H, E, \widetilde{E}, \widetilde{D}, U$ and the operator $A$ as in Sect. 5. Let, moreover, $D\left([a, b] ; \mathbb{R}^{d}\right)$ denote the space of $\mathbb{R}^{d}$-valued càdlàg functions on the interval $[a, b]$, equipped with the supremum norm and set

$$
O=\mathbb{R}^{d} \times\left\{\varphi \in D\left([-T, 0) ; \mathbb{R}^{d}\right): \exists \lim _{s \rightarrow 0^{-}} \varphi(s) \in \mathbb{R}^{d}\right\}
$$

Then $E \subset O \subset H$ and $O$ is isomorphic to $D\left([-T, 0] ; \mathbb{R}^{d}\right)$. Through (36) we can define $M_{T}$ as a map from $O$ to $D\left([0, T] ; \mathbb{R}^{d}\right)$.

For a given continuous path $\gamma_{t} \in C\left([0, t] ; \mathbb{R}^{d}\right)$ consider the stochastic differential equation in $\mathbb{R}^{d}$

$$
\mathrm{d} y(s)=b\left(s, y_{s}\right) \mathrm{d} s+\sigma\left(s, y_{s}\right) \mathrm{d} W(s), \quad s \in[t, T], \quad y_{t}=\gamma_{t},
$$

and assume that $b$ and $\sigma$ are regular enough for Eq. (47) to have a continuous solution $y^{\gamma_{t}}$ (compare Sect. 5.3).

Choose $f \in C_{b}^{2, \alpha}\left(D\left([0, T] ; \mathbb{R}^{d}\right)\right)$ and define

$$
\begin{aligned}
& \Phi: O \rightarrow \mathbb{R} \\
& \Phi(x)=f\left(M_{T} x\right) .
\end{aligned}
$$

If the operators $B, C$ are defined from $b$ and $\sigma$ as in (38), (39), we can consider the infinite-dimensional Kolmogorov backward equation 


$$
\left\{\begin{array}{l}
\frac{\partial V}{\partial t}(t, x)+\langle D V(t, x), A x+B(t, x)\rangle+\frac{1}{2} \operatorname{Tr}_{\mathbb{R}^{d}}\left(C(s, x) C(s, x)^{*} D^{2} V(t, x)\right)=0 \\
V(T, \cdot)=\Phi
\end{array}\right.
$$

We call Eq. (49) the path-dependent Kolmogorov backward equation associated to the triple ( $b, \sigma, f)$. A classical solution $V$ to Eq. (49) uniquely identifies a path-dependent functional $v$, which is given by

$$
v\left(t, \gamma_{t}\right)=V\left(t, L^{t} \gamma_{t}\right)
$$

Since $L^{t} \gamma_{t} \in \widetilde{E}$ if and only if $\gamma_{t} \in C\left([0, t] ; \mathbb{R}^{d}\right)$, it is an immediate consequence of Theorem 5 that for every $t$ the function $v(t, \cdot)$ given by (50) is uniquely determined on $C\left([0, t] ; \mathbb{R}^{d}\right)$.

Therefore, our uniqueness result for path-dependent Kolmogorov equations takes the following form.

Theorem 6 Let $b$ and $\sigma$ such that Eq. (47) has a continuous solution for every $t \in$ $[0, T]$ and every $\gamma_{t} \in C\left([0, t] ; \mathbb{R}^{d}\right)$. Then, for any $f \in C_{b}^{2, \alpha}\left(D\left([0, T] ; \mathbb{R}^{d}\right)\right)$, any path-dependent functional $v$ such that the function $V(t, x)=v\left(t, M_{t} x\right)$ is a classical solution to the path-dependent Kolmogorov backward equation associated to $(b, \sigma, f)$ is uniquely determined on $C\left([0, t] ; \mathbb{R}^{d}\right)$.

Proof Set $\tilde{v}\left(t, \gamma_{t}\right)=V\left(t, L^{t} \gamma_{t}\right)$. By the definition of $M_{T}$, the restriction of $\Phi$ to $E$ belongs to $C_{b}^{2, \alpha}(E)$. Thanks to the assumptions on $b$ and $\sigma$, for any $\gamma_{t} \in C\left([0, t] ; \mathbb{R}^{d}\right)$ there exists a mild solution $X^{t, x}$ to Eq. (46) with $x=L^{t} \gamma_{t} \in \widetilde{E}$. By Propositions 3 and $2 X^{t, x}$ takes values in $\widetilde{E}$ and has continuous paths with respect to the topology of E.

Therefore, we can apply Theorem 5 , and hence, $\tilde{v}$ is uniquely determined on $C\left([0, t] ; \mathbb{R}^{d}\right) ;$ but for any $\gamma_{t} \in C_{t}$

$$
v\left(t, \gamma_{t}\right)=v\left(t, M_{t} L^{t} \gamma_{t}\right)=V\left(t, L^{t} \gamma_{t}\right)=\tilde{v}\left(t, \gamma_{t}\right)
$$

Remark 5 For $\gamma_{t} \in C\left([0, t] ; \mathbb{R}^{d}\right)$ and $x=\left(\gamma_{t}(t), L^{t} \gamma_{t}\right)^{T}$ the process $X^{t, x}$ in the previous proof is given by

$$
X^{t, x}(s)=L^{s} y_{s}^{\gamma_{t}} .
$$

Therefore, if $v$ and $V$ are as above we have, by the definition of solution and Theorem 5 , that

$$
\begin{aligned}
v\left(t, \gamma_{t}\right) & =V\left(t, L^{t} \gamma_{t}\right) \\
& =\mathbb{E}\left[\Phi\left(X^{t, x}(T)\right)\right] \\
& =\mathbb{E}\left[f\left(M_{T} X^{t, x}(T)\right)\right] \\
& =\mathbb{E}\left[f\left(M_{T} L^{T} y_{T}^{\gamma_{t}}\right)\right] \\
& =\mathbb{E}\left[f\left(y_{T}^{\gamma_{t}}\right)\right] .
\end{aligned}
$$


This is what one would expect to be the solution to a Kolmogorov equation with terminal condition $f$ associated (in some sense) to the SDE (47).

Notice that the extension of $\gamma_{t}$ introduced by the operator $L^{t}$ is arbitrary; nevertheless it does not play any role in the path-dependent Kolmogorov equation since $B$ and $C$ are defined using $M_{t}$; compare Remark 2.

\subsection{Another Example on Kolmogorov Equations}

We try to identify a class of functions solving virtually a Kolmogorov type equations. The inspiration comes from [8], Section 9.9, see also [5], Theorem 3.5 for a variant.

Let $N \in \mathbb{N}, g_{1}, \ldots, g_{N} \in B V([0, T])$. We set $g_{0}=1$. We define by $\Sigma(t)$ the $(N+1) \times(N+1)$ matrix

$$
\Sigma_{i j}(t):=\int_{t}^{T} g_{i}(s) g_{j}(s) \mathrm{d} s
$$

We suppose that $\Sigma(t)$ is invertible for any $0 \leq t<T$. We denote by

$$
p_{t}(x)=\frac{1}{(2 \pi)^{\frac{N+1}{2}} \sqrt{\operatorname{det} \Sigma(t)}} \exp \left(-\frac{1}{2} x^{T} \Sigma^{-1}(t) x\right)
$$

the Gaussian density with covariance $\Sigma(t)$, for $t \in[0, T), x \in \mathbb{R}^{N+1}$. Let $f$ : $\mathbb{R}^{N+1} \rightarrow \mathbb{R}$ be a continuous function with polynomial growth. We set

$$
\widehat{g}_{j}(s)=g_{j}(s+T)
$$

$0 \leq j \leq N, s \in[-T, 0]$. We consider $H: C([-T, 0]) \rightarrow \mathbb{R}$ defined by

$$
H(\eta)=f\left(\eta(0), \int_{[-T, 0]} \widehat{g}_{1} d \eta, \ldots, \int_{[-T, 0]} \widehat{g}_{N} d \eta\right)
$$

where

$$
\int_{[-T, 0]} \widehat{g}_{i} d \eta:=\widehat{g}_{i}(0) \eta(0)-\int_{[-T, 0]} \eta d \widehat{g}_{i} .
$$

To simplify, let us assume $g_{i}$ continuous.

We define $\mathscr{U}:[0, T] \times \mathbb{R} \times C([-T, 0]) \rightarrow \mathbb{R}$ by

$$
\mathscr{U}(t, x, \psi)=\tilde{\mathscr{U}}\left(t, x, \int_{[-T, 0]} g_{1}(\cdot+t) d \psi, \ldots, \int_{[-T, 0]} g_{N}(\cdot+t) d \psi\right),
$$

where $\tilde{\mathscr{U}}:[0, T] \times \mathbb{R} \times \mathbb{R}^{N} \rightarrow \mathbb{R}$ is motivated by the following lines. 
We consider the martingale

$$
M_{t}=E\left[h \mid \mathscr{F}_{t}\right]
$$

where (with $\left.\widehat{W}_{s}=W_{s+T}, s \in[-T, 0]\right)$,

$$
h=H(\widehat{W})=f\left(W_{T}, \int_{0}^{T} g_{1}(s) \mathrm{d} W_{s}, \ldots, \int_{0}^{T} g_{N}(s) \mathrm{d} W_{s}\right) .
$$

We proceed by a finite-dimensional analysis.

We remind that $\widehat{g}_{j}(s)=g_{j}(s+T)$. We evaluate more specifically the martingale $M$. We get

$$
M_{t}=\tilde{\mathscr{U}}\left(t, W_{t}, \int_{0}^{t} g_{1}(s) \mathrm{d} W_{s}, \ldots, \int_{0}^{t} g_{N}(s) \mathrm{d} W_{s}\right),
$$

where

$$
\begin{aligned}
\tilde{\mathscr{U}} & \left(t, x, x_{1}, \ldots, x_{N}\right) \\
& =E\left[f\left(x+W_{T}-W_{t}, x_{1}+\int_{t}^{T} g_{1} \mathrm{~d} W, \ldots, x_{N}+\int_{t}^{T} g_{N} \mathrm{~d} W\right)\right] \\
& =\int_{\mathbb{R}^{N+1}} f\left(x+\xi_{0}, x_{1}+\xi_{1}, \ldots, x_{N}+\xi_{N}\right) p_{t}(\xi) d \xi \\
& =\int_{\mathbb{R}^{N+1}} f\left(\xi_{0}, \xi_{1}, \ldots, \xi_{N}\right) p_{t}\left(x-\xi_{0}, x_{1}-\xi_{1}, \ldots, x_{N}-\xi_{N}\right) d \xi_{0} d \xi_{1} \ldots d \xi_{N} .
\end{aligned}
$$

By inspection we can show, see also [8], that $\tilde{\mathscr{U}} \in C^{1,2}\left([0, T) \times \mathbb{R}^{N+1}\right)$

$$
\begin{aligned}
\partial_{t} \tilde{\mathscr{U}}+\frac{1}{2} \sum_{i, j=0}^{N} \Sigma_{i j}(t) \frac{\partial^{2} \tilde{\mathscr{U}}}{\partial x_{i} \partial x_{j}} & =0 \\
\tilde{\mathscr{U}}(T, x) & =f(x),
\end{aligned}
$$

where $x=\left(x_{0}, x_{1}, \ldots, x_{N}\right)$. This can be done via the property of the density kernel $(t, \xi) \mapsto p_{t}(\xi)$ and classical integration theorems. We set $\mathscr{U}:[0, T] \times \mathbb{R} \times$ $C([-T, 0]) \rightarrow \mathbb{R}$ as in (51).

Proposition 4 Let $C^{2}:=C^{2}([-T, 0])$. The map $\mathscr{U}$ has the following properties:

(i) $\mathscr{U} \in C^{0,2,0}$

(ii) $\mathscr{U} \in C^{1,2,1}\left([0, T] \times \mathbb{R} \times C^{2}\right)$

(iii) the map

$$
(t, x, \psi) \longmapsto \mathscr{A}(\mathscr{U})(t, x, \psi):=\partial_{t} \mathscr{U}(t, x, \psi)+\left\langle D^{\psi} \mathscr{U}(t, x, \psi), \psi^{\prime}\right\rangle
$$

extends continuously on $[0, T] \times \mathbb{R} \times C([-T, 0])$ to an operator still denoted by $\mathscr{A}(\mathscr{U})(t, x, \psi)$ 
(iv)

$$
\partial_{t} \mathscr{U}+\mathscr{A}(\mathscr{U})+\frac{1}{2} \partial_{x x}^{2} \mathscr{U}=0 .
$$

Proof (i) Obvious.

(ii) We evaluate the different derivatives for $(t, x, \psi) \in[0, T] \times \mathbb{R} \times C^{2}$. We get from (51)

$$
\begin{aligned}
\partial_{t} \mathscr{U}(t, x, \psi)= & \partial_{t} \widetilde{\mathscr{U}}\left(t, x, \int_{[-t, 0]} g_{1}(\cdot+t) d \psi, \ldots, \int_{[-t, 0]} g_{N}(\cdot+t) d \psi\right) \\
& +\sum_{j=1}^{N} \partial_{j} \widetilde{\mathscr{U}}\left(t, x, \int_{[-t, 0]} g_{1}(\cdot+t) d \psi, \ldots, \int_{[-t, 0]} g_{N}(\cdot+t) d \psi\right) \\
& \times \frac{d}{\mathrm{~d} t} \int_{[-t, 0]} g_{j}(\cdot+t) d \psi
\end{aligned}
$$

Now we observe that

$$
\begin{aligned}
\frac{d}{\mathrm{~d} t} \int_{[-t, 0]} g_{j}(\cdot+t) d \psi & =\frac{d}{\mathrm{~d} t} \int_{[-t, 0]} g_{j}(\xi) \psi^{\prime}(\xi-t) d \xi \\
& =g_{j}(t) \psi^{\prime}(0)-\int_{[-t, 0]} g_{j}(\xi) \psi^{\prime \prime}(\xi-t) d \xi \\
& =\int_{[-t, 0]} \psi^{\prime}(\xi-t) g_{j}^{\prime}(d \xi)
\end{aligned}
$$

(remark that, without restriction of generality, we can take $\left.g_{j}(0)=0\right)$. Now we calculate

$$
\begin{aligned}
& \left\langle D^{\psi} \mathscr{U}(t, x, \psi), \psi^{\prime}\right\rangle \\
& =\sum_{j=1}^{N} \partial_{j} \tilde{\mathscr{U}}\left(t, x, \int_{[-t, 0]} g_{1}(\cdot+t) d \psi, \ldots, \int_{[-t, 0]} g_{N}(\cdot+t) d \psi\right) \\
& \quad \times\left\langle D^{\psi} \int_{[-t, 0]} g_{j}(\cdot+t) d \psi, \psi^{\prime}\right\rangle .
\end{aligned}
$$

Now the application

$$
\begin{aligned}
\psi & \longmapsto \int_{[-t, 0]} g_{j}(\cdot+t) d \psi=\int_{[-t, 0]} g_{j}(\xi+t) \psi^{\prime}(\xi) d \xi \\
& =-\int_{[-t, 0]} d \psi(\xi) \int_{(\xi+t, 0]} d g_{j}(l)=-\int_{(0, t]} d g_{j}(l) \int_{[l-t, 0)} d \psi(\xi) \\
& =-\int_{(0, t]} d g_{j}(l) \psi(l-t)
\end{aligned}
$$


has to be differentiated in the direction $\psi^{\prime}$. Taking into account (53), (54), (55), it follows that

$$
\begin{array}{rl}
\partial_{t} & \mathscr{U}(t, x, \psi)+\left\langle D^{\psi} \mathscr{U}(t, x, \psi), \psi^{\prime}\right\rangle \\
\quad=\partial_{t} \tilde{\mathscr{U}}\left(t, x, \int_{[-t, 0]} g_{1}(\cdot+t) d \psi, \ldots, \int_{[-t, 0]} g_{N}(\cdot+t) d \psi\right)
\end{array}
$$

for every $\psi \in C^{2}$. On the other hand by (52) it follows that $\mathscr{U} \in C^{1,2,1}([0, T] \times$ $\left.\mathrm{R} \times C^{2}\right)$.

(iii) By (56), for $(t, x, \psi) \in[0, T] \times \mathbb{R} \times C^{2}$, we get

$$
\mathscr{A}(\mathscr{U})=\partial_{t} \tilde{\mathscr{U}}\left(t, x, \int_{[-t, 0]} g_{1}(\cdot+t) d \psi, \ldots, \int_{[-t, 0]} g_{N}(\cdot+t) d \psi\right)
$$

(iv) This claim follows by inspection, taking into account (52).

Acknowledgements Open access funding provided by Institute of Science and Technology (IST Austria). The second named author benefited partially from the support of the "FMJH Program Gaspard Monge in Optimization and Operations Research" (Project 2014-1607H). He is also grateful for the invitation to the Department of Mathematics of the University of Pisa. The third named author is grateful for the invitation to ENSTA.

Open Access This article is distributed under the terms of the Creative Commons Attribution 4.0 International License (http://creativecommons.org/licenses/by/4.0/), which permits unrestricted use, distribution, and reproduction in any medium, provided you give appropriate credit to the original author(s) and the source, provide a link to the Creative Commons license, and indicate if changes were made.

\section{References}

1. Bensoussan, A., Da Prato, G., Delfour, M.C., Mitter, S.K.: Representation and Control of Infinite Dimensional Systems. Systems \& Control: Foundations \& Applications. Birkhäuser, Boston (1992)

2. Cerrai, S., Da Prato, G.: A basic identity for Kolmogorov operators in the space of continuous functions related to RDEs with multiplicative noise. Ann. Probab. 42(4), 1297-1336 (2014)

3. Cont, R., Fournié, D.A.: Change of variable formulas for non-anticipative functional on path space. J. Funct. Anal. 259, 1043-1072 (2010)

4. Cont, R., Fournié, D.A.: Functional Ito calculus and stochastic integral representation of martingales. Ann. Probab. 41(1), 109-133 (2013)

5. Cosso, A., Russo, F.: Functional Itô versus Banach space stochastic calculus and strict solutions of semilinear path-dependent equations. Infin. Dimens. Anal. Quantum Probab. Relat. Top. (to appear)

6. Da Prato, G., Jentzen, A., Röckner, M.: A Mild Itô Formula for SPDE's. arXiv:1009.3526 [Math.PR] (2010)

7. Da Prato, G., Zabczyk, J.: Stochastic Equations in Infinite Dimensions. Encyclopedia of Mathematics and Its Applications. Cambridge University Press, Cambridge (1992)

8. Di Girolami, C., Russo, F.: Infinite Dimensional Stochastic Calculus Via Regularization. Preprint HAL-INRIA. http://hal.archives-ouvertes.fr/inria-00473947/fr/ (2010)

9. Di Girolami, C., Russo, F.: Generalized covariation and extended Fukushima decomposition for Banach space-valued processes. Applications to windows of Dirichlet processes. Infin. Dimens. Anal. Quantum Probab. Relat. Top. 15(2), 1250,007, 50 (2012). doi:10.1142/S0219025712500075

10. Di Girolami, C., Russo, F.: Generalized covariation for Banach space valued processes, Itô formula and applications. Osaka J. Math. 51, 729-783 (2014)

11. Dupire, B.: Functional Itô calculus. Portfolio Research Paper 2009-04, Bloomberg (2009) 
12. Fabbri, G., Russo, F.: Infinite dimensional weak Dirichlet processes and convolution type processes. Stoch. Process. Its Appl. (to appear). doi:10.1016/j.spa.2016.06.010

13. Flandoli, F., Zanco, G.: An infinite-dimensional approach to path-dependent Kolmogorov equations. Ann. Probab. 44(4), 2643-2693 (2016). doi:10.1214/15-AOP1031

14. Masiero, F.: Regularizing properties for transition semigroups and semilinear parabolic equations in Banach spaces. Electron. J. Probab. 12, 387-419 (2007)

15. van Neerven, J., Veraar, M., Weis, L.: Stochastic integration in Banach spaces-a survey. In: Stochastic Analysis: A Series of Lectures, Progress in Probability, vol. 68, pp. 297-332. Springer (2015)

16. Zanco, G.: Infinite-dimensional methods for path-dependent stochastic differential equations. Ph.D. Thesis, Università di Pisa (2015) 